\title{
The ROSAT point spread functions and associates
}

\author{
F.G. Boese \\ Max-Planck-Institut für extraterrestrische Physik, D-85740 Garching, Germany \\ e-mail: gub@mpe.mpg.de
}

Received September 30; accepted November 17, 1999

\begin{abstract}
The ROSAT point spread functions for the instruments XRT-PSPC, XRT-HRI, (XUV telescope)-WFC in pointing mode as well as the ROSAT survey point spread functions are documented. A series of plots exhibits the point spread function models. Commands dedicated and calibration tables related to point spread functions are explained. The internet addresses for the accompanying documentation are given.
\end{abstract}

Key words: X-ray: general, space vehicles: instrumentation: detectors

\section{Introduction}

This article is meant as technical reference for questions related to the point spread functions of the instruments of the ROSAT satellite. The latter are fully and in a unified form presented. So, the point spread function densities, the cumulative distributions in both ROSAT observation modes are given. Spread measures derived from the point spread functions such as FWHM and medians are introduced. The underlying assumptions for the point spread function models are laid open. Model limitations are mentioned.

Section 2 introduces the instruments of the ROSAT satellite. Section 3 explains general features of the point spread functions. In three subsections the point spread functions of the two different X-ray detectors and the extreme UV detector are presented. In a fourth subsection, the ROSAT survey point spread function is considered. At the expense of a slight redundance, the exposition is complete at the detector level. All material belonging to a detector is presented in the respective subsection. The effect of the physical attitude jitter or imprecise attitude data on the point spread function is treated in Sect. 4. The conclusions are collected in the final Sect. 5. An Appendix,

Send offprint requests to: F.G. Boese via gub@mpe.mpg.de
Sect. 6, contains point spread function related aids provided by the data analysis system EXSAS.

\section{The imaging instruments}

The ROSAT satellite was launched on July 1, 1990 into a circular orbit with an inclination of $53^{\circ}$ and an altitude of $580 \mathrm{~km}$. The last observation was made on December 18, 1998 and the official end of the ROSAT mission is February 12, 1999.

The larger of the two telescopes on board ROSAT is a grazing incidence X-ray telescope (XRT) consisting of four nested Wolter type I mirrors. The X-ray imaging telescope (XRT) was used in combination with either the Position Sensitive Proportional Counter (PSPC) or the High Resolution Imager (HRI) as focal detector.

Two observation modes, pointed observation and survey observation, were possible. In pointed observation the instrument's optical axis remained nominally fixed relative to the observation target within the observation interval. In survey observation mode, the optical axis (as unit vector) describes nominally circles in a plane perpendicular to the direction Earth-Sun.

The ROSAT All-Sky Survey (RASS) was performed with the PSPC detector. ROSAT carried the Wide Field Camera (WFC), a third imaging instrument with a separate extreme ultraviolet imaging telescope (XUV) coaligned with the XRT. This telescope is comprised of a nest of three grazing incidence Wolter-Schwarzschild type I mirrors. For further details, see the instrument description in Trümper $(1990,1991)$ and the instrument specific papers.

\section{Point spread functions and associates}

For certain purposes of spatial analysis, the point spread function (PSF) of the instrument in operation during the observation is of paramount significance.

The PSF is defined to be the (normalized) photon distribution in the focal plane caused by a celestial X-ray 
point source at infinite distance. Parameters are thereby the direction of the X-ray point source relative to the instrument's optical axis and the energy of the incoming photons.

More technically, the photon distribution is conceived as (the density of) a probability distribution $p$ in the detector plane. The implied random variable is the random event of the incidence of one photon in a small neighbourhood around a given point $\mathbf{x}$ in the detector plane. The variables and parameters of $p$ are explained in detail in the sequel.

Analytical representations for the point spread functions of the ROSAT imagers XRT-PSPC, XRT-HRI, and XUV-WFC have been deduced by detailed estimations from in-flight data as well as from pre-launch calibration data.

For the PSPC, the PSF model adopted, its physical justification and the data sets used in the PSF estimation, ground calibration data and in-flight data are described in a series of three articles by Hasinger et al. (1992, 1993, 1994). The ROSAT mirror assembly is documented by Aschenbach (1988). See also the ROSAT spacecraft and instrumentation description in Trümper (1990) or Trümper (1991). The interested reader is referred to these original documents. In case of the HRI, the documentation is similar, see the report by David et al. (1999). The pertinent WFC documents are those by Barstow (1990), Brunner et al. (1993), Sansom (1990), Wells (1990), Sansom (1991), Willingale (1988) and again Trümper (1990) or Trümper (1991).

Imagine momentarily an ideal imaging system with complete focusing in a focal plane ${ }^{1}$ and without stochastic influences. Then, in the geometrical ray approach, all photons having the same energy and coming from the same spatial direction strike the detector focal plane at a certain point, s, called the source position. In a real imaging system, incomplete focusing as well as stochastic imaging processes caused by the micro-roughness of the mirror ${ }^{2}$ and the detector physics are inevitable, and the assumed source point widens to an extended point spread function (PSF), viewed here as a two dimensional probability density over the detector plane $\mathbf{R}^{2}$ (in general) closely around the hypothetical source point $\mathbf{s}$. Suppose only photons of the same energy, $E$, and coming from the same spatial direction arrive at the detector plane. Then $p \mathrm{~d} A$ is the expected photon count fraction falling into the area element $\mathrm{d} A$ around the point $\mathbf{x}$ in the detector plane. The quantity $p$ has thus the dimension ${ }^{3}$ (Photon Counts)/Area.

\footnotetext{
1 The PSPC has no "real" focal plane. An incoming photon releases an avalanche of electrons in a certain depth in the detector chamber. The higher the photon energy, the deeper the formation of the electron shower.

2 The deviation of the mirror geometry from the ideal one causes a major part of the mirror defocusing.

3 The distinction between true photons and counted photons ("events") is not made here.
}

The general position, $\mathbf{x}$, in the detector plane will be described by means of two polar coordinate systems. The first, the optical axis system $\{\mathcal{O} ; \epsilon, \theta\}$, has its pole at the trace point, $\mathcal{O}$, of the optical axis of the mirror-detector system in the detector plane. The point $\mathbf{s}$ is referred to this system so that ${ }^{4} \epsilon:=|\mathbf{s}-\mathcal{O}|$, henceforth called off-axis angle, is the angular distance between the source position $\mathbf{s}$ and the optical axis' trace point $\mathcal{O}$. Further, the azimuthal angle $\theta$ is measured in positive, i.e. counter-clockwise, direction off the positive horizontal axis. The second system, the source system $\{\mathbf{s} ; r, \phi\}$, is a translate of the first one and attached to the source position $\mathbf{s}$, so that $r:=|\mathbf{x}-\mathbf{s}|$ is the source distance. The azimuthal angle $\phi$ of $\mathbf{x}-\mathbf{s}$ is measured in the same way off the related horizontal axis as in the optical axis system. Although known to exist at larger off-axis angles, no azimuthal dependence has been modelled so far. The models to follow (WFC excepted) represent the azimuthally averaged part of the observed PSF $^{5}$. Consequently, the azimuthal angles $\theta, \phi$ do not occur in the parameterization of $p$. The remaining parameters of $p$ are thus the photon energy $E$ and the off-axis angle $\epsilon$. The source distance $r$ is conceived as the variable.

Any ROSAT mirror-detector combination establishes a one-to-one correspondence, the so-called ray-trace relation, between the photon's arrival directions relative to the optical axis, forming the field of view, and the image of the field of view in the detector plane. So, the distances $\epsilon, r$ can be identified with angular distances from the related central positions $\mathcal{O}, \mathbf{s}$ and are thus measured in angular units, namely the off-axis angle $\epsilon$ in $\operatorname{arcmin}$ and $r$ in arcsec - the units of the arguments of the PSF $p$ used together with $E$ in $\mathrm{keV}$.

The obtainment of an estimate, $\hat{\mathbf{s}}$, for the unknown source position $\mathbf{s}$ itself belongs to the tasks of the spatial analysis. Having found $p$, its mode (i.e. peak-) position serves for $\hat{\mathbf{s}}$. The subsequent notation stresses the dependency on the parameters.

According to the above definition, the point spread functions $p$ are normalized so that

$$
\int_{\mathbf{R}^{2}} p(r ; E, \epsilon) r \mathrm{~d} r \mathrm{~d} \phi=1
$$

This means that $p$ is the distribution density for one photon. As introduced above, $r$ in arcsec is the angular distance of the area element $\mathrm{d} A:=r \mathrm{~d} r \mathrm{~d} \phi$ in polar coordinates $r, \phi$ from the source position $\mathbf{s}$ in the detector plane $\mathbf{R}^{2}$. Moreover, $E$ in $\mathrm{keV}$ is the energy of the photon registered and $\epsilon$ in arcmin the angular distance of $\mathbf{s}$ from the trace point $\mathcal{O}$ of the optical axis in the detector plane.

\footnotetext{
The expression " $A:=B$ " or " $B=: A$ " means: $A$ is defined by $B$.

${ }^{5}$ In our usage, the meticulous signification of the term PSF is: analytical model of azimuthally averaged part of the observed PSF.
} 
Besides $p$, the cumulative point spread function, $P$, i.e. the radially and azimuthally integrated $p$,

$P(r ; E, \epsilon):=\int_{0}^{2 \pi} \mathrm{d} \phi \int_{0}^{r} p(\rho ; E, \epsilon) \rho \mathrm{d} \rho$

is of relevance ${ }^{6}$. The quantity $P(r ; E, \epsilon)$ is the fraction of photon counts which is expected within the circle $|\mathbf{x}-\mathbf{s}|=r$ of the detector plane when the photons with energy $E$ hit the detector plane at a distance $\epsilon$ from the optical axis' trace point $\mathcal{O}$. In case of the WFC, the geometry of point spread function is more general; the level curves will be formed by ellipses with the shorter axis in radial direction.

The normalization (1) implies the limiting relation $P(r ; E, \epsilon) \rightarrow 1$ for $r \rightarrow \infty$.

We come to measures of spread for the PSF. The $q$ quantile radius $r_{q}$ is defined implicitly by $q=P\left(r_{q} ; E, \epsilon\right)$, $0 \leq q<1$. So, for $q=1 / 2$ the median $r_{1 / 2}$ is obtained. The diameter $2 r_{1 / 2}$ is also called half-energy-width (HEW) in the context of monochromatic spectra. Associated with $p$ is also the full-width-half-maximum (FWHM) function, $w(E, \epsilon)$, implicitly defined by

$\frac{1}{2}=\frac{p\left(\frac{1}{2} w(E, \epsilon) ; E, \epsilon\right)}{p(0 ; E, \epsilon)}$.

All analytical ROSAT point spread function models decrease with increasing $r$ so that $w(E, \epsilon)$ is uniquely defined.

Several measures of spread of distributions are known and in use. The appropriate choice among them depends on the context. The FWHM characterizes the spread of a PSF density. The median radius $r_{1 / 2}$, defined by $P\left(r_{1 / 2}, E, \epsilon\right)=1 / 2$, is another measure of spread for the cumulative $\mathrm{PSF}^{7}$.

Before describing the PSF in detail, recall again that two ROSAT observation modes were possible, pointed observation and all-sky survey observation. In contrast to survey observation, in most pointed observations a wobbling motion around the nominal pointing direction is carried out in order to lessen the detrimental shadow of the detector window support structure.

In this connection, some alerting words are in order. The attempt to verify the point spread functions given below from event files or images - as they are - may fail.

\footnotetext{
${ }^{6}$ The term encircled energy for $P$ is not used here. In our case photon counts are distributed, not energy. In this article, $p$ and $P$ are the generic symbols for a photon probability density distribution and $P$ for a probability distribution. So, the reader should be prepared to see that $p$ denotes different distributions in different context. For example, $p(r ; E, \epsilon)$ in (6) and in (10) are different.

${ }^{7}$ For two-dimensional circularly symmetric normal distributions, i.e. not azimuthally dependent, the relation $\frac{1}{2} F W H M=$ median is valid. In general, however, both quantities are unrelated.
}

First, recall that the ROSAT attitude error $^{8}$ was specified to be up to 10 arcsec. Any attempt to stay below this specification requires an intimate instrument knowledge. Details of the achieved positional accuracy can be found in Voges et al. (1999).

Secondly, recall that uncorrected remainders of a wobbling motion in saw-tooth form $S[u]$ and a possible systematic or stochastic attitude $\operatorname{drift}^{9} \delta(t)$ may be superimposed to the nominal pointing direction of the telescope after attitude correction for wobbling remainders in the Standard Analysis Software System (SASS). This leads, after mapping onto the detector plane, to the adoption of the non-stationary, i.e. time-dependent, stochastic process model for the source position

$$
\begin{aligned}
& \mathbf{s}(t):=\mathbf{s}_{0}+\mathbf{w} S\left[2 \pi \nu\left(t-t_{0}\right)\right]+\delta(t), \quad t_{1} \leq t \leq t_{2}, \\
& S[u]:= \begin{cases}4\{u\}, & 0 \leq\{u\} \leq 1 / 4, \\
2-4\{u\}, & 1 / 4 \leq\{u\} \leq 3 / 4 \\
4\{u\}-4, & 3 / 4 \leq\{u\} \leq 1\end{cases}
\end{aligned}
$$

in place of the time-independent source position s. In (4), $\{u\}$ denotes the fractional part of the (dimensionless) real number $u$. Moreover, $\mathbf{s}_{0}$ is the unknown true source position and $\mathbf{w}$ the constant wobbling vector with the nominal magnitude $|\mathbf{w}|=3$ arcmin. Deviations from the nominal value are known. After the ROSAT standard data processing, the remaining $|\mathbf{w}|$ is expected to be considerably smaller. At a time $t=t_{0}$ in (or close to) the observation time interval $\left[t_{1}, t_{2}\right]$, the wobbling motion vanishes. The wobbling frequency for ROSAT, $\nu$, has the nominal value $\nu=1 / 402 \mathrm{~Hz}$. Finally, $\delta(t)$ is at best a white noise process but is expected to have normally a systematic and/or stochastic additional drift component motion. The task is to find estimates $\hat{\mathbf{w}}, \hat{\nu}, \hat{t}_{0}, \hat{\delta}(t)$ for the counterparts in (4) based on the observation at hand. Then (4) with these estimates is to be solved for

$\hat{\mathbf{s}}_{0}:=\mathbf{s}(t)-\hat{\mathbf{w}} S\left[2 \pi \hat{\nu}\left(t-\hat{t}_{0}\right)\right]+\hat{\delta}(t)$.

The correction vector $\mathbf{c}(t):=\hat{\mathbf{w}} S\left[2 \pi \hat{\nu}\left(t-\hat{t}_{0}\right)\right]+\hat{\delta}(t)$ is, finally, to be subtracted from the photons arriving at time $t$ in the observation interval $t_{1} \leq t \leq t_{2}$ to the effect that (a) systematic distortions due to wobbling are (greatly) removed and (b) the variances of photon clusters around source positions diminish. Use of attitude data may assist the described de-wobbling and "de-speckling" procedure. The success of this effort may vary from observation to observation. Details, limitations and examples of the sketched method are beyond the scope of this paper.

\footnotetext{
8 Two constitutive parts are to be named; a contribution due to the imprecise attitude reconstruction and a physical part. The latter comes from the temperature dependent misalignment of the axes of star tracker and mirror and other sources.

${ }^{9}$ Systematic drift may be caused by thermal boresight deviation or by angular momenta due to the magnetic coils.
} 
Having done this, a good agreement between the point spread functions estimated that way and the ones below should be reached. The particular observations at hand may not allow a de-speckling. How to account for an attitude drift $\delta(\mathbf{t})$ in such a case will be discussed in Sect. 4 in more detail.

\subsection{The point spread function for the ROSAT XRT-PSPC instrument}

The authors of the three papers by Hasinger et al. (1992, $1993,1994)$ carried out the considerable amount of work related with the estimation of the PSF under consideration.

In the present case, $p:=p(r ; E, \epsilon)$ is parameterized by the photon energy, $E$, and the off-axis angle, $\epsilon$, as a three component additive mixture with energy and off-axis angle dependent mixture proportions $p_{1}(E, \epsilon), p_{2}(E, \epsilon), p_{3}(E)$,

$$
\begin{aligned}
p(r ; E, \epsilon):= & p_{1}(E, \epsilon) \cdot \frac{\mathrm{e}^{-\frac{1}{2}\left(\frac{r}{\sigma(E, \epsilon)}\right)^{2}}}{2 \pi \sigma^{2}(E, \epsilon)}+p_{2}(E, \epsilon) \cdot \frac{\mathrm{e}^{-\frac{r}{R(E)}}}{2 \pi R^{2}(E)} \\
& +p_{3}(E) \cdot m(r ; E), \\
m(r ; E):= & \frac{1}{2 \pi\left[\frac{1}{2} \ln \left[1+\left(\frac{r_{2}}{r_{1}}\right)^{2}\right]+\frac{1}{[\alpha(E)-2]\left[1+\left(\frac{r_{1}}{r_{2}}\right)^{2}\right]}\right]} \\
& \cdot \begin{cases}\frac{1}{r_{1}^{2}(E)+r^{2}}, \\
\frac{1}{r_{1}^{2}(E)+r_{2}^{2}(E)} \cdot\left(\frac{r_{2}(E)}{r}\right)^{\alpha(E)}, & r \geq r_{2}(E) .\end{cases}
\end{aligned}
$$

The first addend in (6) stems from the random process taking place with the generation of primary electrons in the counter. The second term results from the finite penetration depth of the X-ray photons in the counter gas and the diffusion of the electron cloud. The last term is due to the mirror scattering. The estimates for the functions and parameters occuring in (6) are

$$
\begin{aligned}
\sigma(E, \epsilon): & \sqrt{108.7 E^{-0.888}+1.121 E^{6}+0.219 \epsilon^{2.848}}, \\
R(E): & \sqrt{50.61 E^{-1.472}+6.8 E^{5.62}}, \\
r_{1}(E):= & \frac{39.95}{E} \\
r_{2}(E):= & \frac{861.9}{E} \\
\alpha(E):= & 2.119+0.212 E \\
p_{3}(E):= & 0.04 E^{1.43}, \\
p_{2}(E, \epsilon):= & \min \left\{10^{0.639 E+0.052 E^{2}-1.635} \cdot \mathrm{e}^{-\left(\frac{\epsilon}{12}\right)^{2} / 2},\right. \\
& \left.1-p_{3}(E)\right\}, \\
p_{1}(E, \epsilon):= & 1-p_{3}(E)-p_{2}(E, \epsilon) .
\end{aligned}
$$

The units used for $r, E, \epsilon$ are arcsec, $\mathrm{keV}$, arcmin, respectively, and the numbers in (7) have the implied units, e.g. 39.95 arcsec keV in the case of $r_{1}$. Observe that the quotient $r_{2}(E) / r_{1}(E)$ in (6) does not depend on $E$ and is thus written without argument $E$. The above estimate of $p_{3}(E)$ was found by P. Predehl (private communication) and replaces since end 1995 the older estimate $0.075 E^{1.43}$.

The PSPC field of view has a diameter $\approx 2^{\circ}$ so that $r \leq 7200$ arcsec. This, together with the energy range in which the energy dependencies of (10) hold, gives for applications the domain of definition of $p(r ; E, \epsilon)$,

$0 \leq r \leq 7200, \quad 0.07 \leq E \leq 3.0, \quad 0 \leq \epsilon \leq 60$.

Figure 1 shows the radial dependence of the XRT-PSPC

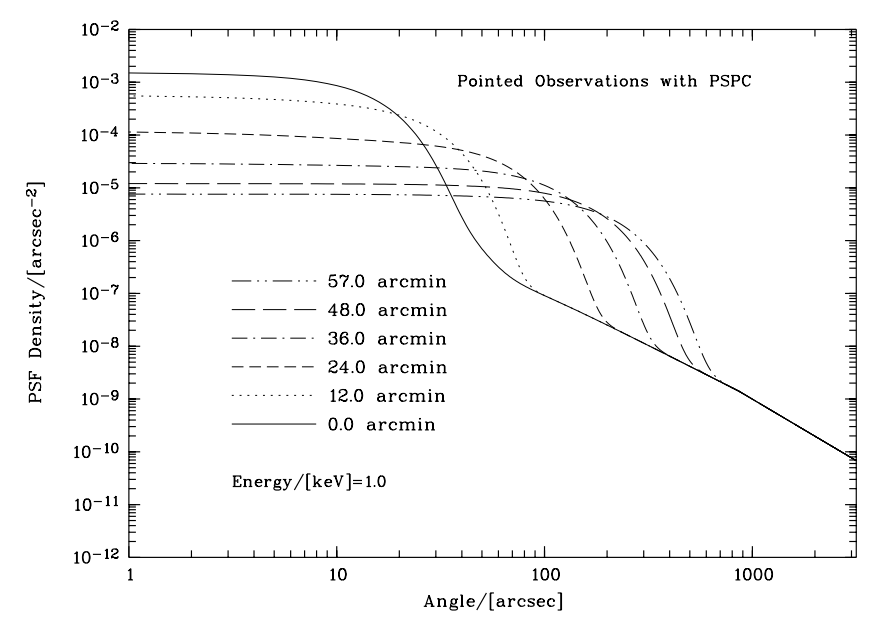

Fig. 1. PSPC Pointing PSF density for $E=1 \mathrm{keV}, \epsilon=0,12$, 24, 36, 48 and 57 arcmin

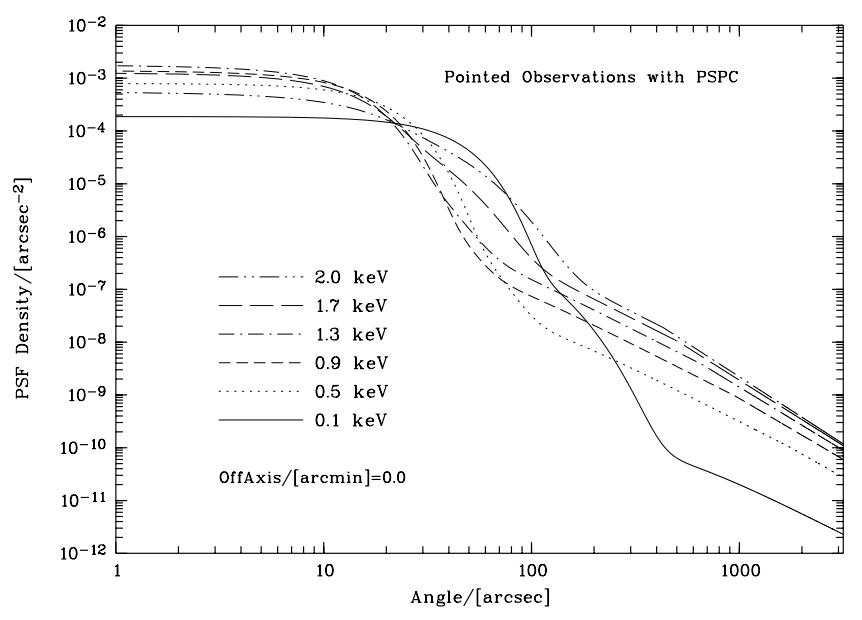

Fig. 2. On-Axis PSPC Pointing PSF density for $E=0.1,0.5$, $0.9,1.3,1.7$ and $2 \mathrm{keV}$ 


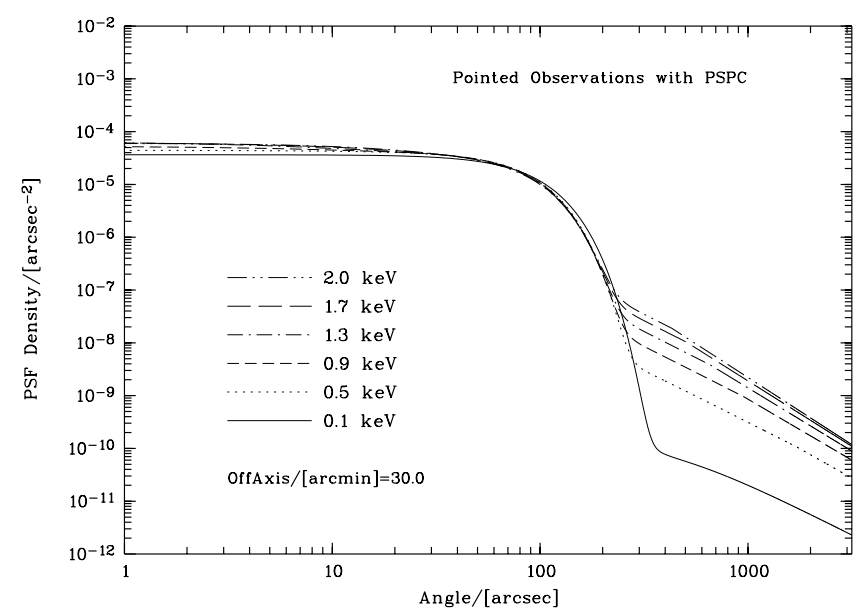

Fig. 3. PSPC Pointing PSF density for $\epsilon=30$ arcmin, $E=0.1$, $0.5,0.9,1.3,1.7$ and $2 \mathrm{keV}$

PSF density $p(r ; E, \epsilon)$ in pointing mode for a photon energy of $1 \mathrm{keV}$ for six different off-axis angles $\epsilon=0(12) 48$ and $57^{10}$ arcmin in the range $0 \leq r \leq 3600$ arcsec in logarithmical scaling of the abscissa and ordinate.

The point spread function becomes wider as the off-axis angle increases. The energy dependence of the on-axis, PSPC pointing PSF is exhibited in Fig. 2 for the energies $E=0.1,0.5,0.9,1.3,1.7$ and $2 \mathrm{keV}$. Figure 2 is supplemented by Fig. 3 showing the PSPC PSF density at a large off-axis angle $\epsilon=$ 30 arcmin and the same photon energies as in Fig. 2, $E=0.1,0.5,0.9,1.3,1.7$ and $2 \mathrm{keV}$.

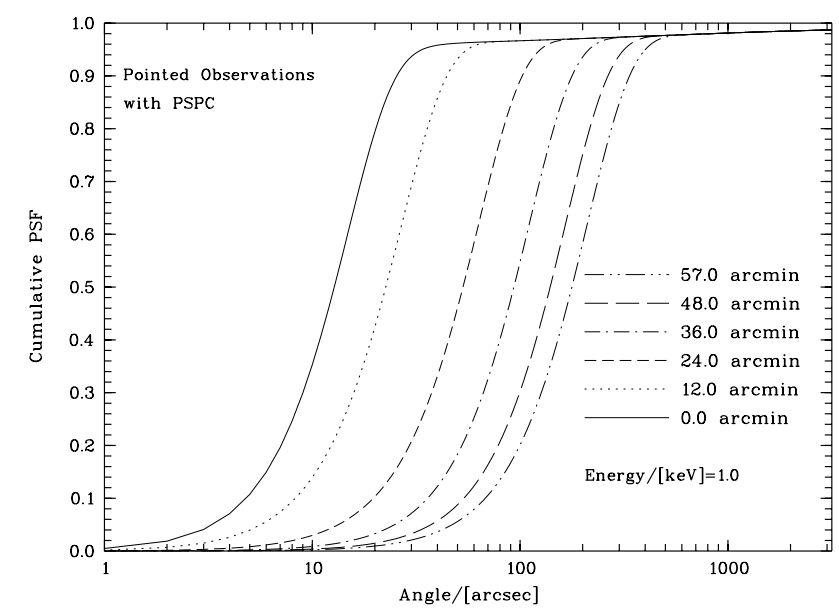

Fig. 4. Cumulative PSPC Pointing PSF at energy $E=1 \mathrm{keV}$ and off-axis angles $\epsilon=0,12,24,36,48$ and 57 arcmin

The cumulative distribution $P(r ; E, \epsilon)$ from (2) belonging to $p(r ; E, \epsilon)$ from (6) with parameters from (7)

10 The expression " $k=a(b) c$ " means: integer variable $k$ runs from $a$ to $c$ in steps of $b$.

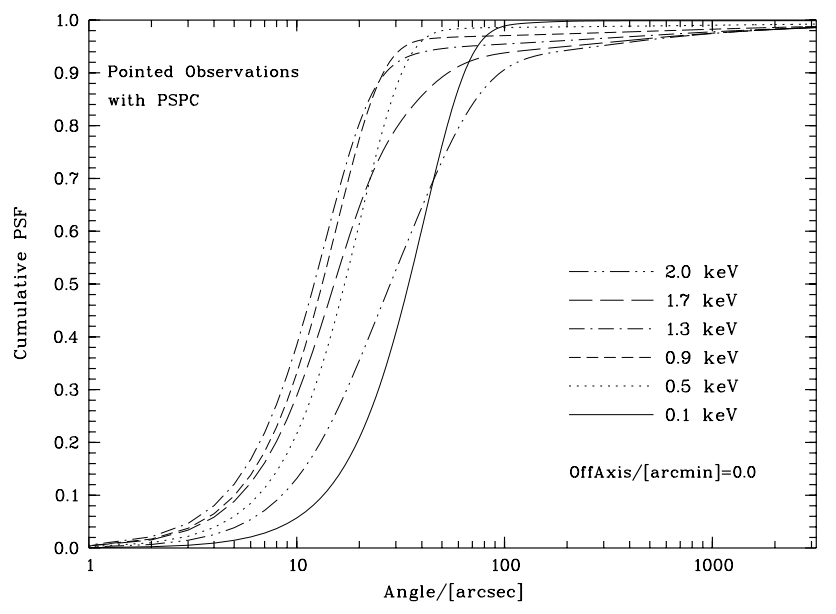

Fig. 5. Cumulative On-Axis PSPC Pointing PSF for energies $E=0.1,0.5,0.9,1.3,1.7$ and $2 \mathrm{keV}$

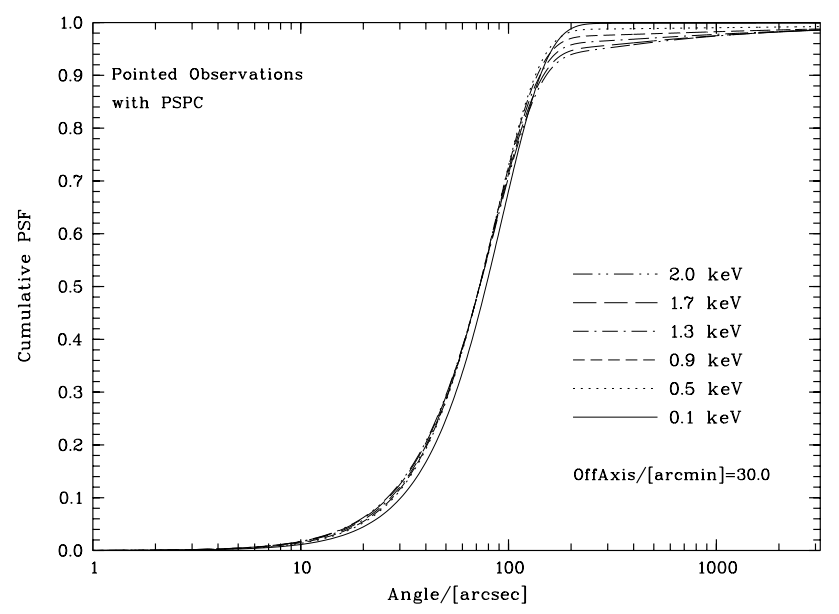

Fig. 6. Cumulative Off-Axis PSPC Pointing PSF at $\epsilon=$ 30 arcmin for energies $E=0.1,0.5,0.9,1.3,1.7,2 \mathrm{keV}$

is found to be

$$
\begin{gathered}
P(r ; E, \epsilon):=p_{1}(E, \epsilon) \cdot\left[1-\mathrm{e}^{\left.-\frac{1}{2}\left(\frac{r}{\sigma(E, \epsilon)}\right)^{2}\right]+}\right. \\
p_{2}(E, \epsilon) \cdot\left[1-\left(1+\frac{r}{R(E)}\right) \cdot \mathrm{e}^{\left.-\frac{r}{R(E)}\right]+}\right. \\
p_{3}(E) \cdot M(r ; E), \\
M(r ; E):=\frac{1}{\frac{1}{2} \ln \left[1+\left(\frac{r_{2}}{r_{1}}\right)^{2}\right]+\frac{1}{[\alpha(E)-2]\left[1+\left(\frac{r_{1}}{r_{2}}\right)^{2}\right]}} \cdot \\
\left\{\begin{array}{l}
\frac{1}{2} \ln \left[1+\left(\frac{r}{r_{1}(E)}\right)^{2}\right], \\
\frac{1}{2} \ln \left[1+\left(\frac{r_{2}}{r_{1}}\right)^{2}\right]+\frac{1-\left(\frac{r_{2}(E)}{r}\right)^{\alpha(E)-2}}{[\alpha(E)-2]\left[1+\left(\frac{r_{1}}{r_{2}}\right)^{2}\right]}, r \geq r_{2}(E) .
\end{array}\right.
\end{gathered}
$$

Figures 4 to 6 display the corresponding cumulative counterparts to Figs. 1 to 3. Thus Fig. 4 shows the ROSAT 
XRT-PSPC cumulative pointing PSF for the same parameters as in Fig. 1, namely, for $\epsilon=0,12,24,36,48$ and 57 arcmin and $E=1 \mathrm{keV}$ in the same range $0 \leq r \leq$ 3600 arcsec in double logarithmical representation.

The larger the off-axis angle, the lower the initial slope at $r=0$. Figure 5 shows the cumulative counterpart to Fig. 2.

The cumulative counterpart of Fig. 3 is Fig. 6 with a large off-axis angle $\epsilon=30$ arcmin and photon energies $E=0.1,0.5,0.9,1.3,1.7,2 \mathrm{keV}$. For further details and reference, consult the articles and documents compiled below.

\subsection{The point spread function for the ROSAT XRT-HRI}

The presently used PSF without the mirror term was determined by David et al. (1999) ${ }^{11}$. The mirror contribution was added by P. Predehl (private communication).

This point spread function is modelled as an additive two-component mixture of a mirror contributions, $p_{\mathrm{M}}$, and a detector component, $p_{\mathrm{D}}$, with energy dependent mixture proportions $D(E), M(E)$,

$$
\begin{aligned}
p(r ; E, \epsilon):= & D(E) \cdot p_{\mathrm{D}}(r ; \epsilon)+M(E) \cdot p_{\mathrm{M}}(r ; E), \\
p_{\mathrm{D}}(r ; \epsilon):= & \frac{1}{2 \pi\left[A_{1} \sigma_{1}^{2}+A_{2} \sigma_{2}^{2}(\epsilon)+A_{3} \sigma_{3}^{2}\right]} \cdot \\
& \left(A_{1} \mathrm{e}^{-\frac{1}{2}\left(\frac{r}{\sigma_{1}}\right)^{2}}+A_{2} \mathrm{e}^{-\frac{1}{2}\left(\frac{r}{\sigma_{2}(\epsilon)}\right)^{2}}+A_{3} \mathrm{e}^{-\frac{r}{\sigma_{3}}}\right), \\
p_{\mathrm{M}}(r ; E):= & \frac{1}{2 \pi\left[\frac{1}{2} \ln \left\{1+\left(\frac{r_{2}}{r_{1}}\right)^{2}\right\}+\frac{1}{[\alpha(E)-2]\left[1+\left(\frac{r_{1}}{r_{2}}\right)^{2}\right]}\right]} \\
& \cdot \begin{cases}\frac{1}{r_{1}^{2}(E)+r^{2}}, & r \leq r_{2}(E), \\
\frac{1}{r_{1}^{2}(E)+r_{2}^{2}(E)} \cdot\left(\frac{r_{2}(E)}{r}\right)^{\alpha(E)}, & r \geq r_{2}(E) .\end{cases}
\end{aligned}
$$

Since PSPC and HRI share the same telescope, the XRT, the functions $m(r, E)$ in $(6)$ and $p_{\mathrm{M}}(r ; E)$ in (10) are identical. The estimates for the parameters in (10) and the function $\sigma_{2}(\epsilon)$ of the $p_{\mathrm{D}}$ component are

$A_{1}:=0.9638, \quad \sigma_{1}:=2.1858$,

$A_{2}:=0.1798, \sigma_{2}(\epsilon):=3.3+0.019 \epsilon-0.016 \epsilon^{2}+0.0044 \epsilon^{3}$ $A_{3}:=0.0009, \quad \sigma_{3}:=31.69$.

The units of $r, E$ and $\epsilon$ in (11) are again arcsec, keV and arcmin, respectively. Since PSPC and HRI share the same telescope mirrors, also the estimates for the parameters

111999 was the year of the latest revision of the report at the time of writing whose history spans some years.
$r_{1}(E), r_{2}(E), \alpha(E)$ of the mirror component $p_{\mathrm{M}}$ in $(10)$ are the same as in (7),

$$
\begin{aligned}
r_{1}(E) & :=\frac{39.95}{E} \\
r_{2}(E) & :=\frac{861.9}{E} \\
\alpha(E) & :=2.119+0.212 E \\
M(E) & :=0.04 E^{1.43} \\
D(E) & :=1-M(E)
\end{aligned}
$$

Notice that the quotient $r_{2}(E) / r_{1}(E)$ does not depend on $E$. Thus the argument $E$ is dropped in (10).

The diameter of the field of view for the HRI is 38 arcmin. This combined with the permissible energy range gives the domain of definition of $p(r ; E, \epsilon)$ as

$0 \leq r \leq 2400, \quad 0.07 \leq E \leq 3.0, \quad 0 \leq \epsilon \leq 20$.

Figure 7 shows the ROSAT XRT-HRI pointing PSF density $p(r ; E, \epsilon)$ for $E=1 \mathrm{keV}$ and off-axis angles $\epsilon=$ $0(4) 16,19$ arcmin in the range $0 \leq r \leq 3600$ arcsec.

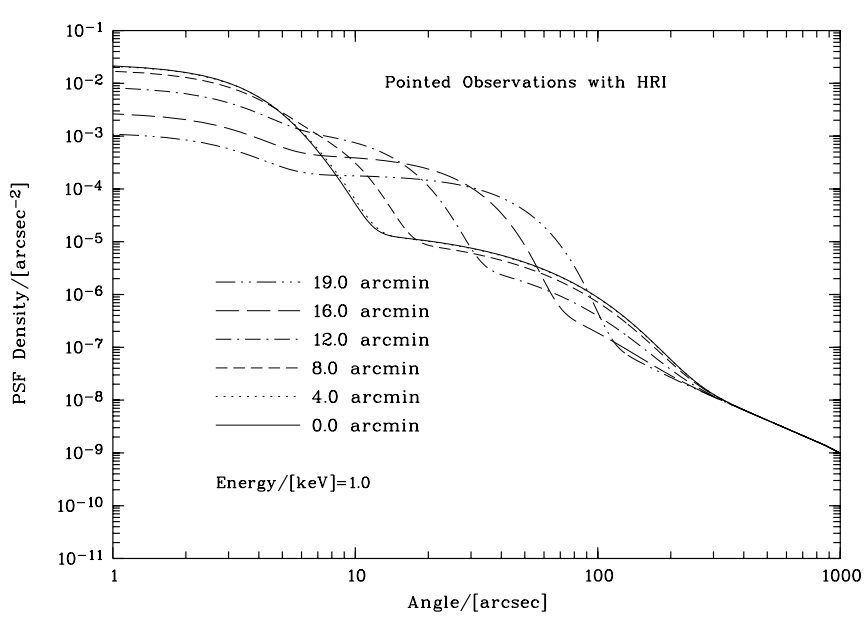

Fig. 7. HRI Pointing PSF density for energy $E=1 \mathrm{keV}$ and off-axis angles $\epsilon=0,4,8,12,16$ and $19 \operatorname{arcmin}$

The point spread function becomes wider as the offaxis angle increases. The energy dependence of the PSF is due to the mirror component and is moderate, as Fig. 8 exhibits.

The curves for $E=0.1,0.5,0.9,1.3,1.7,2 \mathrm{keV}$ are shown. The influence of the term containing $\sigma_{2}(\epsilon)$ is pronounced when the source distance $r$ ranges in $[5,10]$ arcsec, say. Figure 9 extends Fig. 8 in that the HRI PSF density for a large off-axis angle $\epsilon=15$ arcmin and photon energies $E=0.1,0.5,0.9,1.3,1.7$ and $2 \mathrm{keV}$ are shown. 


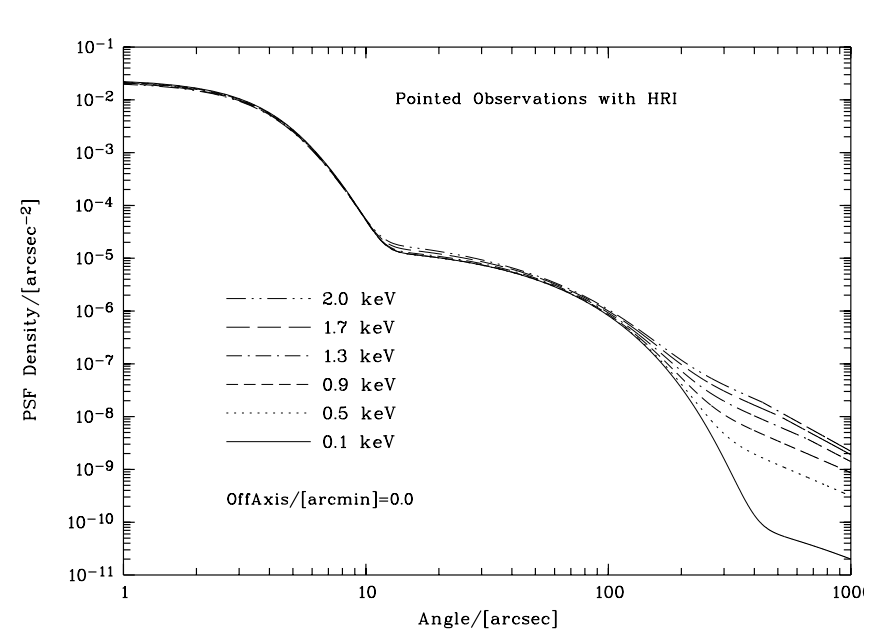

Fig. 8. On-Axis HRI Pointing PSF density for $E=0.1,0.5,0.9$, $1.3,1.7$ and $2 \mathrm{keV}$

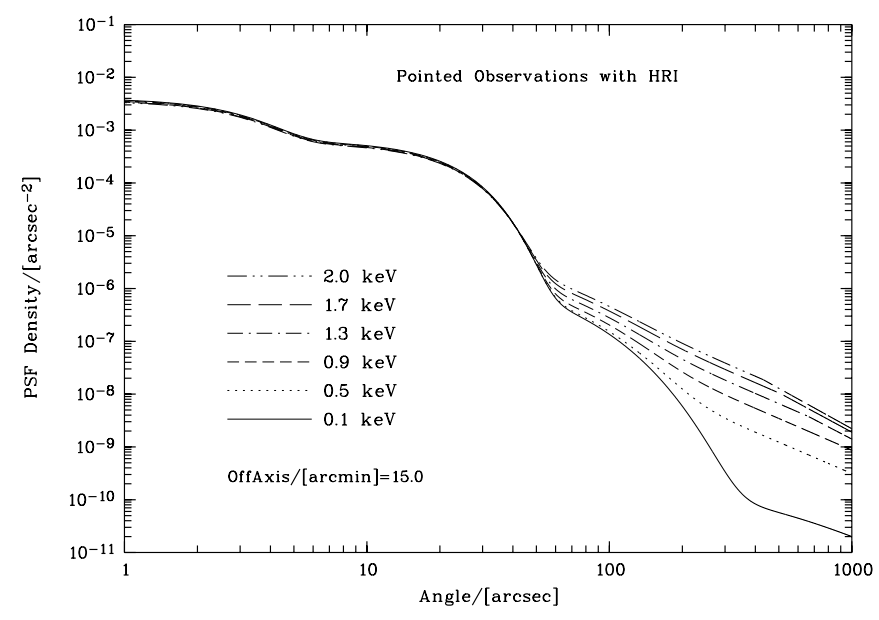

Fig. 9. HRI Pointing PSF density for $\epsilon=15 \operatorname{arcmin}, E=0.1$, $0.5,0.9,1.3,1.7$ and $2 \mathrm{keV}$

The cumulative HRI point spread function is

$$
\begin{aligned}
P(r ; E, \epsilon):= & D(E) \cdot P_{\mathrm{D}}(r ; \epsilon)+M(E) \cdot P_{\mathrm{M}}(r ; E), \\
P_{\mathrm{D}}(r ; \epsilon):= & \frac{A_{1} \sigma_{1}^{2} \cdot\left[1-\mathrm{e}^{\left.-\frac{1}{2}\left(\frac{r}{\sigma_{1}}\right)^{2}\right]}\right.}{A_{1} \sigma_{1}^{2}+A_{2} \sigma_{2}^{2}(\epsilon)+A_{3} \sigma_{3}^{2}} \\
& +\frac{A_{2} \sigma_{2}^{2}(\epsilon) \cdot\left[1-\mathrm{e}^{\left.-\frac{1}{2}\left(\frac{r}{\sigma_{2}(\epsilon)}\right)^{2}\right]}\right.}{A_{1} \sigma_{1}^{2}+A_{2} \sigma_{2}^{2}(\epsilon)+A_{3} \sigma_{3}^{2}} \\
& +\frac{A_{3} \sigma_{3}^{2} \cdot\left[1-\mathrm{e}^{\left.-\left(1+\frac{r}{\sigma_{3}}\right) \frac{r}{\sigma_{3}}\right]}\right.}{A_{1} \sigma_{1}^{2}+A_{2} \sigma_{2}^{2}(\epsilon)+A_{3} \sigma_{3}^{2}}, \\
P_{\mathrm{M}}(r ; E):= & \frac{1}{\frac{1}{2} \ln \left[1+\left(\frac{r_{2}}{r_{1}}\right)^{2}\right]+\frac{1}{[\alpha(E)-2]\left[1+\left(\frac{r_{1}}{r_{2}}\right)^{2}\right]}} .
\end{aligned}
$$

$$
\begin{cases}\frac{1}{2} \ln \left[1+\left(\frac{r}{r_{1}(E)}\right)^{2}\right], & r \leq r_{2}(E), \\ \frac{1}{2} \ln \left[1+\left(\frac{r_{2}}{r_{1}}\right)^{2}\right]+\frac{1-\left(\frac{r}{r_{2}(E)}\right)^{\alpha(E)-2}}{[\alpha(E)-2]\left[1+\left(\frac{r_{1}}{r_{2}}\right)^{2}\right]}, & r \geq r_{2}(E) .\end{cases}
$$

Figure 10 shows the cumulative ROSAT XRT-HRI PSF for the same parameters as in Fig. 7, namely, for $E=1 \mathrm{keV}$ and $\epsilon=0(2) 10$ arcmin, in the same range $0 \leq r \leq 3600$ arcsec. The rule that the initial slope becomes smaller with increasing off-axis angle $\epsilon$ is confirmed in a qualitative way. Figure 11 shows the cumulative counterpart of Fig. 8. Finally, Fig. 12 is the cumulative counterpart of the large off-axis HRI PSF density of Fig. 9 with the same off-axis-angle $\epsilon=15$ arcmin and photon energies $E=0.1,0.5,0.9,1.3,1.7,2 \mathrm{keV}$. For further information and reference see the documents listed below.

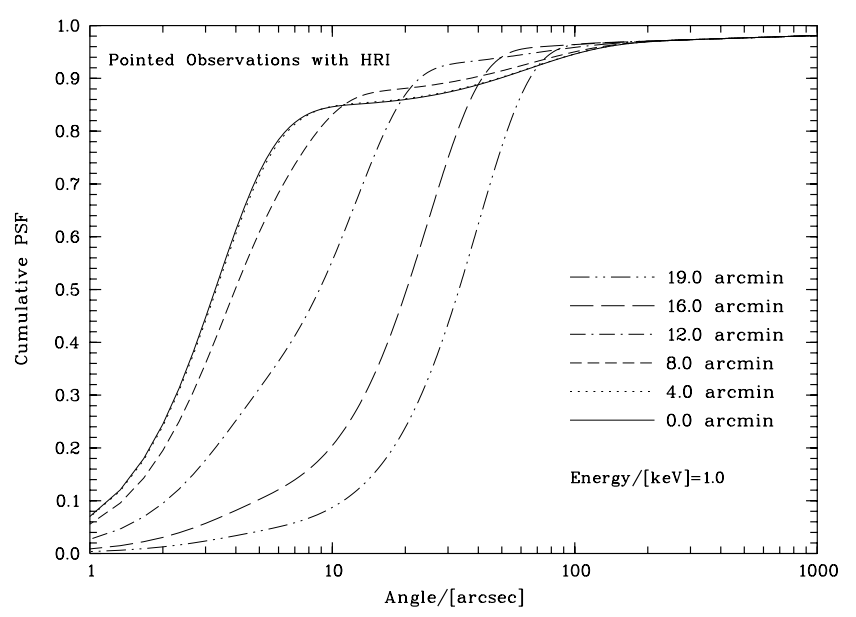

Fig. 10. Cumulative HRI Pointing PSF for $E=1 \mathrm{keV}, \epsilon=0$, 4, 8, 12, 16 and 19 arcmin

\subsection{The point spread function for the ROSAT $X U V-W F C$}

The original work was done by Sansom (1990) and Wells (1990).

As already mentioned, the Wide Field Camera has a separate telescope mirror, and the detector is a microchannel plate detector. The relative large field of view of $5^{\circ}$ diameter results in larger distortions for sources near the border of the field of view. It was found that the geometry of the level curves of the observed PSF changes remarkably with the off-axis angle $\epsilon$. In contrast to the PSPC and HRI detectors, not only the azimuthally averaged part of the observed PSF is modelled. The level curves of the PSPC and HRI PSF models were circles for all off-axis angles. In the WFC case, ellipses replace the circles. 


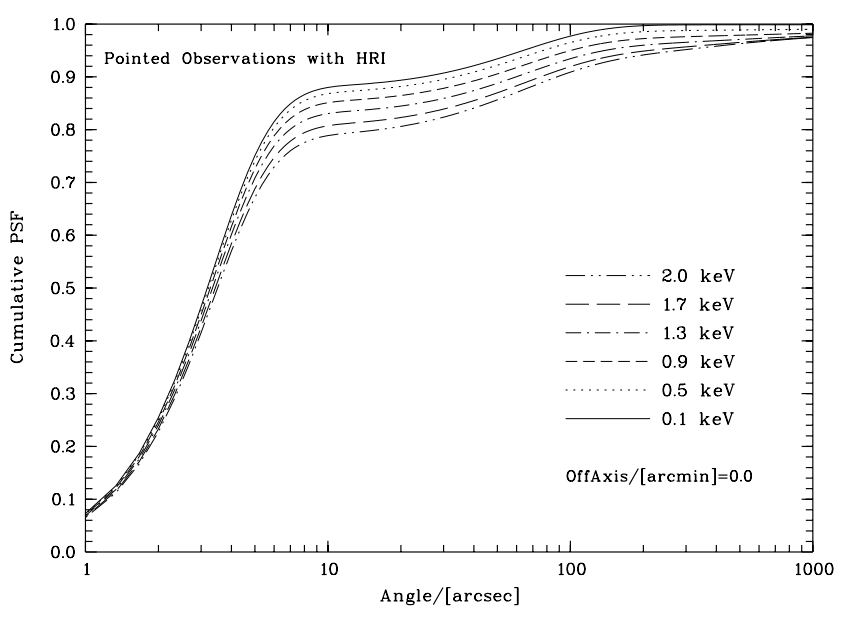

Fig. 11. Cumulative On-Axis HRI Pointing PSF for $E=0.1$, $0.5,0.9,1.3,1.7$ and $2 \mathrm{keV}$

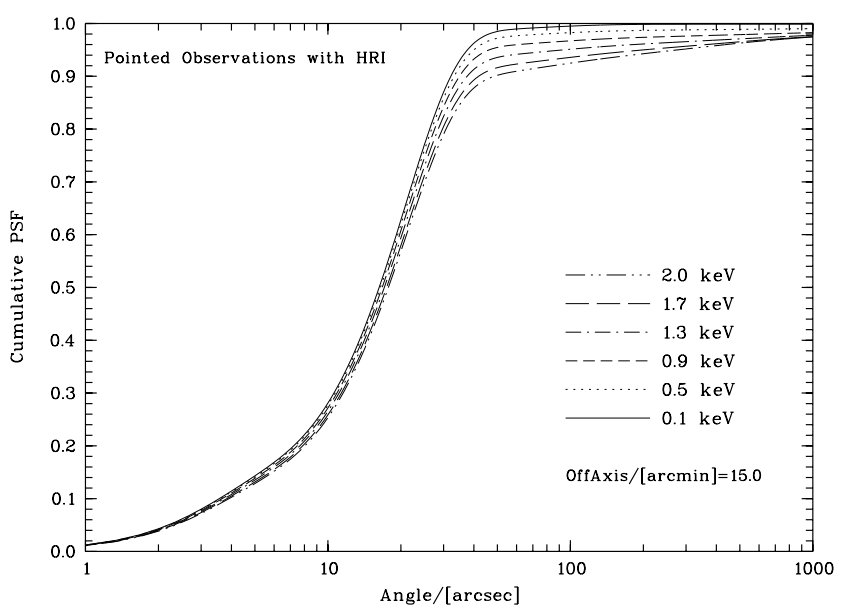

Fig. 12. Cumulative HRI Pointing PSF for $\epsilon=15 \operatorname{arcmin}, E=$ $0.1,0.5,0.9,1.3,1.7$ and $2 \mathrm{keV}$

In order to introduce them, a Cartesian source coordinate system $\{\mathbf{s}, x, y\}$ has to be introduced. The $x$-axis points in the direction of the radius vector from $\mathcal{O}$ to $\mathbf{s}$. Rotating the $x$-axis by $+\pi / 2$ about the source position $\mathbf{s}$ yields the orientation of the $y$-axis. In this coordinate system, the said ellipses have the representation

$\rho(x, y):=\sqrt{\left(\frac{x}{e}\right)^{2}+y^{2}}=r, \quad r \geq 0$,

where

$e:=\{$ length of minor axis $\} /\{$ length of major axis $\} \leq 1$ is a measure of eccentricity ${ }^{12}$. Thus, the $x$-axis is aligned with the minor axis of the ellipse. The relation $e \leq 1$ means a radial squeezing of the PSF distribution.

The ad hoc model of the WFC PSF is an additive mixture of two components, $p_{\mathrm{A}}$ and $p_{\mathrm{B}}$, with energy

\footnotetext{
12 There is no great risk to confound eccentricity $e$ and even
} less $e_{\mathrm{A}}, e_{\mathrm{B}}$ with the mathematical constant $e:=2.71 \ldots$
$E$ and off-axis angle $\epsilon$ dependent mixture proportions $A(E, \epsilon), B(E, \epsilon)$ and with $\alpha(E, \epsilon)>1$,

$$
\begin{aligned}
p(x, y ; E, \epsilon):= & A(E, \epsilon) \cdot p_{\mathrm{A}}(x, y ; E, \epsilon) \\
& +B(E, \epsilon) \cdot p_{\mathrm{B}}(x, y ; E, \epsilon), \\
p_{\mathrm{A}}(x, y ; E, \epsilon):= & \frac{\alpha(E, \epsilon)-1}{\pi \cdot e_{\mathrm{A}}(E, \epsilon) \cdot \sigma_{\mathrm{A}}^{2}(E, \epsilon)} \\
& \cdot \frac{1}{\left[1+\left(\frac{\rho_{\mathrm{A}}(x, y)}{\sigma_{\mathrm{A}}(E, \epsilon)}\right)^{2}\right]^{\alpha(E, \epsilon)}}, \\
p_{\mathrm{B}}(x, y ; E, \epsilon):= & \frac{\mathrm{e}^{-\frac{\rho_{\mathrm{B}}(x, y)}{\sigma_{\mathrm{B}}(E, \epsilon)}}}{2 \pi \cdot e_{\mathrm{B}}(E, \epsilon) \cdot \sigma_{\mathrm{B}}^{2}(E, \epsilon)}, \\
\rho_{\mathrm{C}}(x, y):= & \sqrt{\left(\frac{x}{e_{\mathrm{C}}(E, \epsilon)}\right)^{2}+y^{2}}, \quad C \in\{A, B\}, \\
A(E, \epsilon):= & \frac{1}{1+b(E, \epsilon)}, \\
B(E, \epsilon):= & \frac{b(E, \epsilon)}{1+b(E, \epsilon)} .
\end{aligned}
$$

Again, the units of $x, y, E$ and $\epsilon$ are arcsec, arcsec, $\mathrm{keV}$ and arcmin, respectively. The six functions $\sigma_{\mathrm{A}}(E, \epsilon), e_{\mathrm{A}}(E, \epsilon)$, $\sigma_{\mathrm{B}}(E, \epsilon), e_{\mathrm{B}}(E, \epsilon), \alpha(E, \epsilon)$ and $b(E, \epsilon)$ in $(16)$ are obtained by iterated one-dimensional linear interpolation (or extrapolation) with respect to off-axis angle $\epsilon$ and energy $E$ from the corresponding estimates from (17) estimated at five discrete off-axis angles $\epsilon_{j}, j=1(1) 5$, arcmin and at two energies, $E_{1}=0.0454 \mathrm{keV}$ and $E_{2}=0.1834 \mathrm{keV}$,

$$
\begin{aligned}
& \left(\begin{array}{c}
\epsilon \\
\sigma_{\mathrm{A}} \\
e_{\mathrm{A}} \\
\sigma_{\mathrm{B}} \\
e_{\mathrm{B}} \\
\alpha \\
b
\end{array}\right)=\left(\begin{array}{ccccc}
0.0 & 37.8 & 69.0 & 98.4 & 129.6 \\
21.84 & 35.46 & 42.90 & 75.72 & 104.16 \\
1.0 & 0.98 & 0.93 & 0.83 & 0.67 \\
144.0 & 144.0 & 144.0 & 144.0 & 144.0 \\
1.0 & 1.0 & 1.0 & 1.0 & 1.0 \\
1.43 & 1.37 & 1.42 & 1.55 & 1.47 \\
0.255 & 0.0 & 0.0 & 0.0 & 0.0
\end{array}\right), \\
& \left(\begin{array}{c}
\epsilon \\
\sigma_{\mathrm{A}} \\
e_{\mathrm{A}} \\
\sigma_{\mathrm{B}} \\
e_{\mathrm{B}} \\
\alpha \\
b
\end{array}\right)=\left(\begin{array}{cccccc}
0.0 & 37.8 & 69.0 & 98.4 & 129.6 \\
29.10 & 40.02 & 52.62 & 79.62 & 121.50 \\
1.0 & 0.92 & 0.88 & 0.77 & 0.75 \\
148.32 & 148.32 & 148.32 & 148.32 & 148.32 \\
1.0 & 1.0 & 1.0 & 1.0 & 1.0 \\
1.35 & 1.34 & 1.41 & 1.43 & 1.54 \\
0.265 & 0.0 & 0.0 & 0.0 & 0.0
\end{array}\right) .
\end{aligned}
$$

The upper estimate matrix in (17) belongs to the lower energy $E_{1}$, the lower one to the higher $E_{2}$. Notice that $b(E, \epsilon)>0$ only for the on-axis case $\epsilon=0$ in (17). This setting was caused by the insufficient amount of data available at the time of PSF analysis (Sansom 1990). This applies also to $e_{\mathrm{B}}$.

Denote by $\epsilon_{j}, j=1(1) 5$, the five off-axis angles in the first row of the matrices in (17) in increasing order. Let $q_{k}$ be the parameter of the vectors in $(17)$ from the $(k+1)$ th 
row, $k=1(1) 6$, and $q_{k}\left(E_{l}, \epsilon_{j(\epsilon)}\right)$ the matrix entry at energy $E_{l}$ and off-axis angle $\epsilon_{j}$. Then interpolation with respect to $\epsilon$ followed by that with respect to $E$ based on the estimates of (17) yields the continuous parameter functions for $k=1(1) 6$ as well as $l=1,2$

$$
\begin{aligned}
q_{k}(E, \epsilon):= & q_{k}\left(E_{1}, \epsilon\right) \\
& +\frac{q_{k}\left(E_{2}, \epsilon\right)-q_{k}\left(E_{1}, \epsilon\right)}{E_{2}-E_{1}} \cdot\left(E-E_{1}\right), \\
q_{k}\left(E_{l}, \epsilon\right):= & q_{k}\left(E_{l}, \epsilon_{j(\epsilon)}\right) \\
& +\frac{q_{k}\left(E_{l}, \epsilon_{j^{\prime}(\epsilon)}\right)-q_{k}\left(E_{l}, \epsilon_{j(\epsilon)}\right)}{\epsilon_{j^{\prime}(\epsilon)}-\epsilon_{j(\epsilon)}} \cdot\left(\epsilon-\epsilon_{j(\epsilon)}\right), \\
j(\epsilon):= & \max _{\epsilon_{j} \leq \epsilon} j, \\
j^{\prime}(\epsilon):= & \begin{cases}j(\epsilon)+1, & j(\epsilon)<5, \\
4, & j(\epsilon)=5 .\end{cases}
\end{aligned}
$$

The six functions $\sigma_{\mathrm{A}}(E, \epsilon)$ to $b(E, \epsilon)$ are thus formally defined for all $\epsilon \geq 0, E \geq 0$ but the inequalities

$$
\begin{aligned}
\sigma_{\mathrm{A}}(E, \epsilon) & >0, \quad \sigma_{\mathrm{B}}(E, \epsilon)>0, \\
0 & <e_{\mathrm{A}}(E, \epsilon), e_{\mathrm{B}}(E, \epsilon)<1, \\
1 & <\alpha(E, \epsilon), \quad 0<\beta(E, \epsilon)
\end{aligned}
$$

must be satisfied.

The diameter of the field of view of the WFC is with $\approx 2.5^{\circ}$ in zoom mode and $\approx 5^{\circ}$ without zoom. This, together with the energy range gives for applications the domain of definition of $p(r ; E, \epsilon)$ as

$0 \leq r \leq 18000, \quad 0.017 \leq E \leq 0.210, \quad 0 \leq \epsilon \leq 150$.

The energy interval from (20) corresponds to the $10 \%$ falloff value of the appropriate WFC filter, i.e. the one for the highest energy. The analysis of the system of inequalities (19) shows that all inequalities from (19) are everywhere satisfied in the domain (20). The region $\alpha(E, \epsilon)>1$ defines for $\epsilon \geq 98.4$ in $(E, \epsilon)$-plane the hyperbolic region

$(\epsilon-118.105)(0.1034-E)<11.327$.

The set (21) contains the domain of definition (20). The arc of the boundary of (21) connecting the point $(0.017,249.14)$ with $(0.0416,300)$ forms the curvilinear boundary arcs of the domain (21). So, the domain (20) in the $(E, \epsilon)$-plane is fully contained in $(21)$.

Figure 13 shows the ROSAT XUV-WFC pointing PSF density $p(r ; E, \epsilon)$ for $E=150 \mathrm{eV}$ at the off-axis angles $\epsilon:=k$ arcmin, $k=0,28,56,84,112$ and 133, in the range $0 \leq r \leq 7200$ arcsec in the radial cross-section along the major axis, i.e. for $y=0$ with the UV filter.

The difference to the cross-section along the major axis, i.e. for $x=0$, is so small that no changes are visible at the scale of Fig. 13. Therefore, no plot of the transversal profile is shown. Figure 14 shows the energy dependence

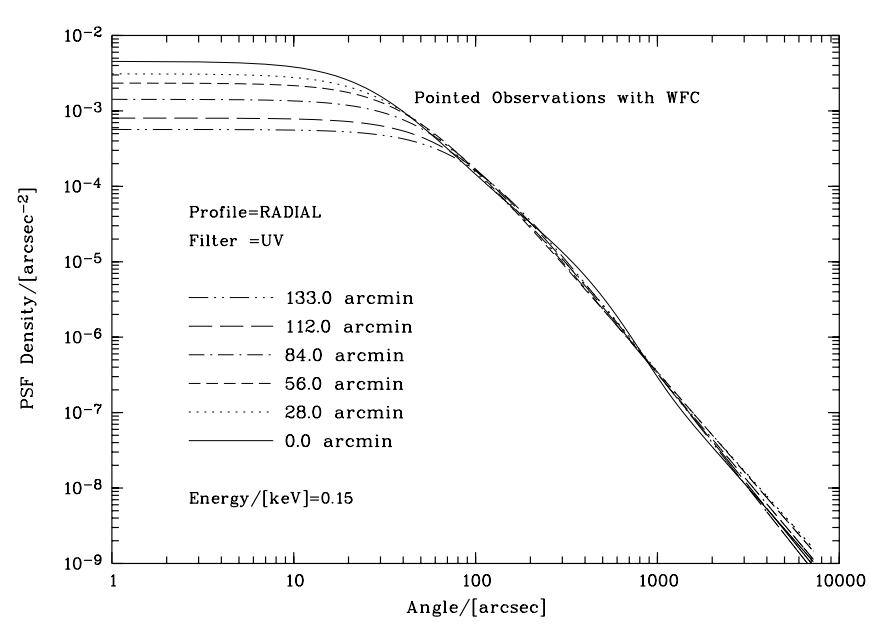

Fig. 13. WFC Pointing PSF density for $E=0.15 \mathrm{keV}, \epsilon=0$, 28, 56, 84, 112 and 133 arcmin, radial cross-section, UV filter

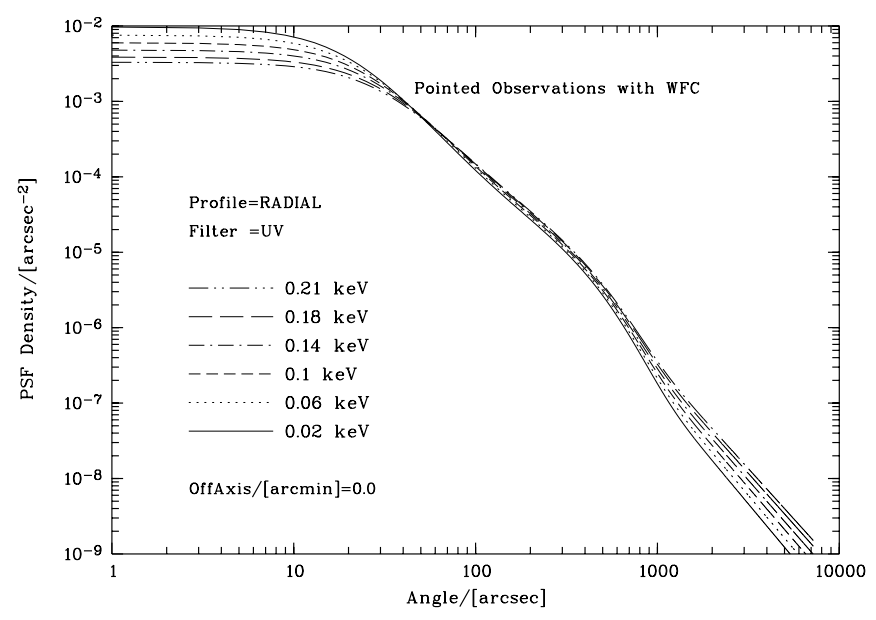

Fig. 14. WFC Pointing PSF density for $\epsilon=0$ arcmin, $E=0.02$, $0.06,0.1,0.14,0.18$ and $0.21 \mathrm{keV}$, radial cross-section, UV filter

of the on-axis WFC density in radial cross-section with the UV filter, as in Fig. 13.

The cumulative pointing point spread function is conveniently defined in the present case by

$P(r ; E, \epsilon):=\int_{\rho_{\mathrm{A}}(x, y) \leq r} p(x, y ; E, \epsilon) \mathrm{d} x \mathrm{~d} y$.

A good approximation and upper bound for $P$ under the data from (17) is the fully explicit expression with $\alpha(E, \epsilon)>1$

$$
\begin{aligned}
& P(r ; E, \epsilon):= A(E, \epsilon) \cdot P_{\mathrm{A}}(r ; E, \epsilon) \\
&+B(E, \epsilon) \cdot P_{\mathrm{B}}(r ; E, \epsilon), \\
& P_{\mathrm{A}}(r ; E, \epsilon):=1-\frac{1}{\left[1+\left(\frac{r}{\sigma_{\mathrm{A}}(E, \epsilon)}\right)^{2}\right]^{\alpha(E, \epsilon)-1}}, \\
& P_{\mathrm{B}}(r ; E, \epsilon):=1-\left(1+\frac{r}{\sigma_{\mathrm{B}}(E, \epsilon)}\right) \mathrm{e}^{-\frac{r}{\sigma_{\mathrm{B}}(E, \epsilon)}} .
\end{aligned}
$$


The $P_{\mathrm{A}}$ component in $(23)$ is exact, and $P_{\mathrm{B}}$ is a sufficiently precise approximation for small off-axis angles $0<\epsilon<37.8$ arcmin and again exact for the remaining off-axis angles $\epsilon \geq 37.8$.

The exact expression for $P_{\mathrm{B}}$ allows the representation

$$
\begin{aligned}
P_{\mathrm{B}}(r ; E, \epsilon):= & 1-\frac{1}{2 \pi} \int_{0}^{2 \pi}\left(1+\frac{r}{\sigma_{\mathrm{B}}(E, \epsilon) \rho(\phi ; E, \epsilon)}\right) \\
& \mathrm{e}^{-\frac{r}{\sigma_{\mathrm{B}}(E, \epsilon) \rho(\phi ; E, \epsilon)}} \mathrm{d} \phi \\
= & 1-\frac{2}{\pi} \int_{0}^{\pi / 2}\left(1+\frac{r}{\sigma_{\mathrm{B}}(E, \epsilon) \rho(\phi ; E, \epsilon)}\right) \\
& \mathrm{e}^{-\frac{r}{\sigma_{B}(E, \epsilon) \rho(\phi ; E, \epsilon)}} \mathrm{d} \phi, \\
\rho(\phi ; E, \epsilon):= & \sqrt{1+\cos ^{2}(\phi) \cdot\left(\frac{e_{B}^{2}(E, \epsilon)}{e_{A}^{2}(E, \epsilon)}-1\right) .}
\end{aligned}
$$

The stable numerical evaluation of $P_{\mathrm{B}}$, as used for the EXSAS command (37), poses no problem. For the estimates from (17), it follows the enclosure

$1-\left(1+\frac{r e_{\mathrm{A}}(E, \epsilon)}{\sigma_{\mathrm{B}}(E, \epsilon) e_{\mathrm{B}}(E, \epsilon)}\right) \mathrm{e}^{-\frac{r e_{\mathrm{A}}(E, \epsilon)}{\sigma_{\mathrm{B}}(E, \epsilon) e_{\mathrm{B}}(E, \epsilon)}} \leq P_{\mathrm{B}}(r ; E, \epsilon)$,

$P_{\mathrm{B}}(r ; E, \epsilon) \leq 1-\left(1+\frac{r}{\sigma_{\mathrm{B}}(E, \epsilon)}\right) \mathrm{e}^{-\frac{r}{\sigma_{\mathrm{B}}(E, \epsilon)}}$.

The rightmost expression in (25) is that of (23). Figure 15 shows the cumulative ROSAT XUV-WFC pointing PSF for the same parameters as in Fig. 13, namely for $E=$ $150 \mathrm{eV}$ and $\epsilon=28,56,84,112$ and 133 arcmin in the same range $0 \leq r \leq 600$ arcsec with the exact $P_{\mathrm{B}}$ from (24).

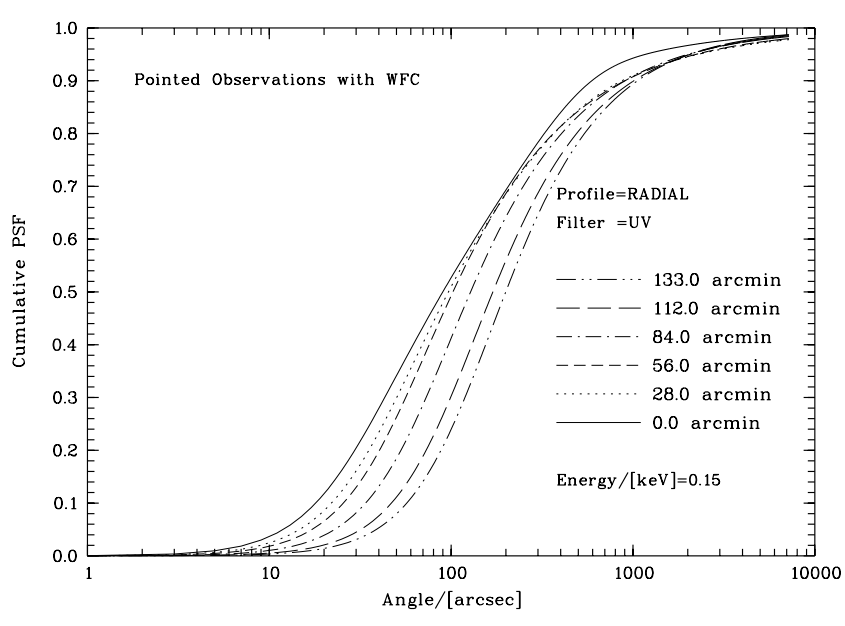

Fig. 15. Cumulative WFC Pointing PSF for $E=0.15 \mathrm{keV}, \epsilon=$ $0,28,56,84,112,133$ arcmin, radial cross-section, UV Filter

The differences to the approximation to $P_{\mathrm{B}}$ from (23) are so small that no deviations are visible at the scale of Fig. 15.

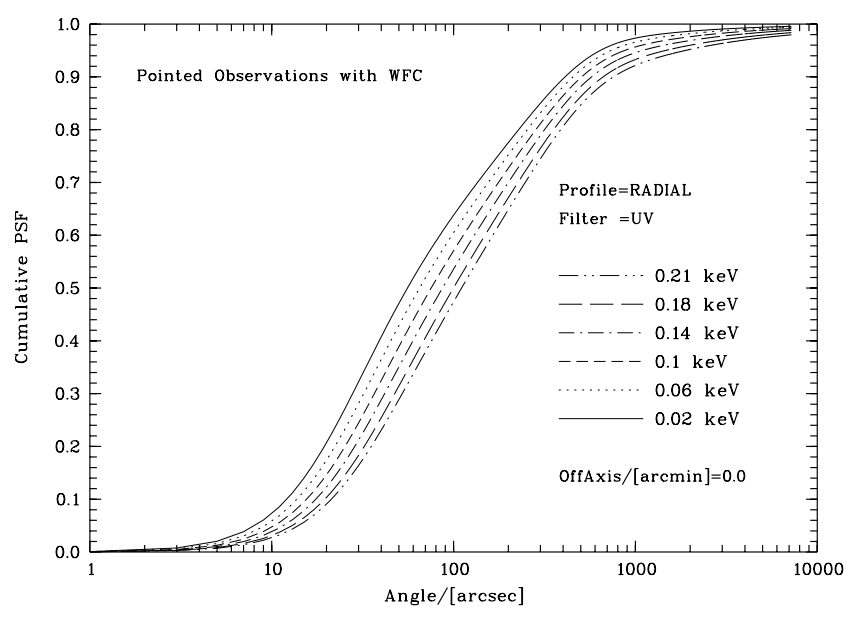

Fig. 16. Cumulative on-axis WFC Pointing PSF for $E=0.02$, $0.06,0.1,0.14,0.18$ and $0.21 \mathrm{keV}$, radial cross-section, UV filter

The component $p_{\mathrm{A}}$ in (16) is sometimes called a Moffat distribution, (see Moffat 1969, Eq. (7)). It is a generalization of the so called King distribution (King 1983, Eq. (10)).

For further details and reference, consult the related documents below.

\subsection{The ROSAT survey point spread function}

Following the trace curve of a celestial point source in the detector's field of view while in survey mode explains that the survey point spread function is a weighted mean with respect to the off-axis angle $\epsilon$ of the corresponding PSF in effect in pointed observation mode.

We will make the following modelling assumptions:

1. The detector's field of view is a circular disk of radius $\epsilon_{0}$ arcmin in the detector plane with the trace point of the optical axis as centre;

2. The scanning angular velocity in survey motion is constant in the field of view;

3. The distance, $\epsilon_{\mathrm{s}}$ arcmin, between consecutive scanning tracks is small $\left(0 \leq \epsilon_{\mathrm{s}} \leq 4 \operatorname{arcmin}\right)$, and we assume the limiting case $\epsilon_{\mathrm{s}} / \epsilon_{0}=0$.

In an observation under analysis, not all scanning tracks may be present. In critical cases, the use of the attitude file frees from accepting assumption 2. Similarly, one can use the more correct weighting distribution corresponding to the distance $\epsilon_{\mathrm{s}}$ actually used in survey motion.

Critical cases in the above sense exist. In a neighbourhood of 1 degree latitude of the ecliptic poles, assumption 3 is violated. Assumption 2 is not obeyed in fields in which the survey observation was interrupted due to the earth's radiation belts or due to the South Atlantic Anomaly.

Based on the above assumptions, the EXSAS implementation of the survey PSF density and the cumulative 
survey PSF with vignetting correction for the PSPC detector will be described and represented in this section.

\subsubsection{The vignetting corrected ROSAT survey point spread function}

The reflectivity of the gold surface decreases with increasing incidence angle on the reflective surface. The projection of the gold-coated reflective viewable area in direction perpendicular to the infalling bundle of X-rays multiplied by the reflectivity is called effective area. It decreases with increasing off-axis angle $\epsilon$. This energy dependent degradation of the mirror assembly is termed vignetting degradation, or in short, vignetting. All previous EXSAS PSFs were not vignetting corrected.

Let $A(E, \epsilon)$ be the effective area function of the mirrordetector system under consideration. Then

$V(E, \epsilon):=\frac{A(E, \epsilon)}{A(E, 0)}$

is the energy and off-axis angle dependent vignetting function for the mirror-detector unit in operation. In EXSAS, $A(E, \epsilon)$ for the detector PSPC_C with which the survey was performed is represented, by the calibration table EXSAS_CAL:effarea_pspcc.tbl with entries for 729 nonequidistant energy values in the interval [0.0713,3.005] $\mathrm{keV}$ and $\epsilon=0(5) 55,57.5$ and 60 . Then the vignetting corrected PSF density, $p^{\mathrm{V}}$, in pointing mode observation is

$p^{\mathrm{V}}(r ; E, \epsilon):=V(E, \epsilon) \cdot p(r ; E, \epsilon)$.

Taking $p^{\mathrm{V}}$ instead of $p$ in (28) leads to the definition for the vignetting corrected survey PSF density, $p_{\mathrm{S}}^{\mathrm{V}}$

$p_{\mathrm{S}}^{\mathrm{V}}(r ; E):=\frac{\int_{0}^{\epsilon_{0}} p(r ; E, \epsilon) V(E, \epsilon) 2 \epsilon \mathrm{d} \epsilon}{\int_{0}^{\epsilon_{0}} V(E, \epsilon) 2 \epsilon \mathrm{d} \epsilon}$.

Figure 17 shows the PSPC vignetting function $V(E, \epsilon)$ for fourteen off-axis angles $\epsilon=0(5) 55,57.5,60$ arcmin in the energy range $[0,3] \mathrm{keV}$. The fact that $V(E, \epsilon)$ decreases in $\epsilon$ allows the identification of the off-axis angle in Fig. 17.

In EXSAS exists yet another table, EXSAS_CAL:vignet_pspc.tbl, with vignetting values. It has entries for the pulse-height values Amplitude = 1(1)300 and the off-axis angles $\epsilon=0(5) 55,57.5$ and 60 . Since it is based on amplitudes rather than energies, it is not to be used for $V(E, \epsilon)$. The survey PSF density is shown in Fig. 18 for the energies $E=0.1,0.5,0.9,1.3,1.7$ and $2.0 \mathrm{keV}$.

The cumulative survey PSF

$P_{\mathrm{S}}^{\mathrm{V}}(r ; E):=2 \pi \int_{0}^{r} p_{\mathrm{S}}^{\mathrm{V}}(\rho ; E) \rho \mathrm{d} \rho$.

is shown in Fig. 19 for the energies $E=0.1,0.5,0.9,1.3$, 1.7 and $2.0 \mathrm{keV}$.

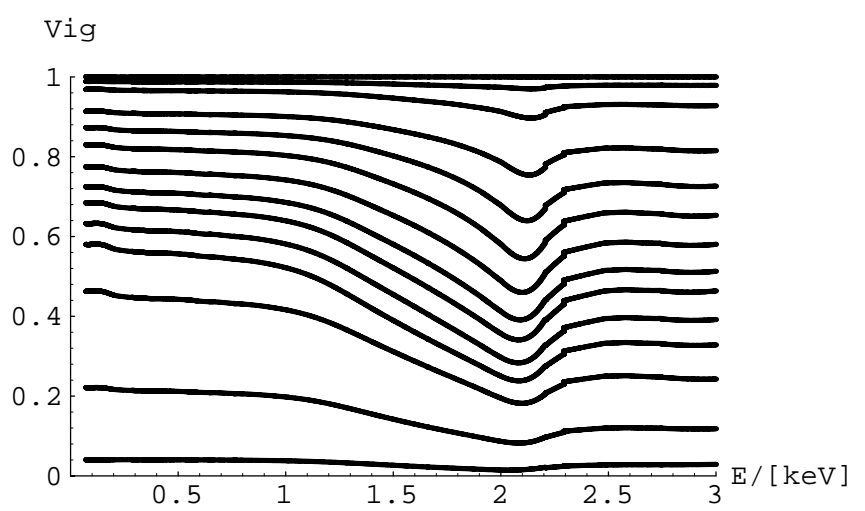

Fig. 17. PSPC vignetting function, Vig, for $\epsilon=0(5) 55,57.5$ and 60 arcmin (top to bottom) in the energy range $[0,3] \mathrm{keV}$

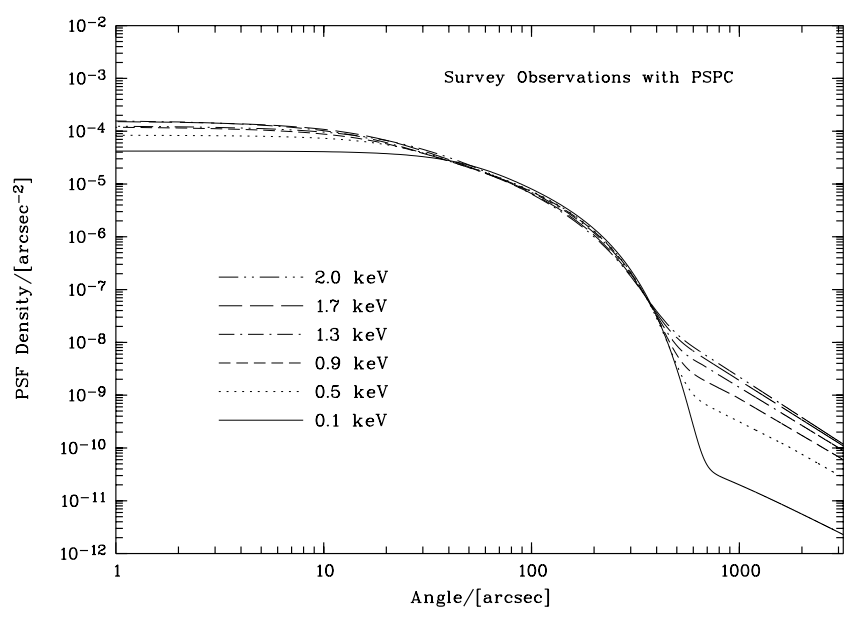

Fig. 18. PSPC Survey PSF density for $E=0.1,0.5,0.9,1.3$, $1.7,2.0 \mathrm{keV}$

\subsection{Survey point spread function based on a Gauss approximation of the PSF}

For the sake of a fast function evaluation, a Gauss approximation of the pointed mode PSPC PSF is used in the EXSAS Maximum Likelihood Source Estimation,

$$
\begin{aligned}
p_{\mathrm{G}}(r ; E, \epsilon) & :=\frac{1}{2 \pi \sigma^{2}(E, \epsilon)} \mathrm{e}^{-\frac{1}{2}\left(\frac{r}{\sigma(E, \epsilon)}\right)^{2}} \\
\sigma(E, \epsilon) & :=\sqrt{108.7 E^{-0.888}+1.21 E^{6}+0.219 \epsilon^{2.848}}
\end{aligned}
$$

Figure 20 shows the PSPC Survey PSF density, $p_{\mathrm{S}}^{\mathrm{G}}(r ; E)$, based on the Gauss approximation $p_{\mathrm{G}}(r ; E)$ as defined in (30) in the interval $[1,3600]$ arcsec for the energies $E=0.1,0.5,0.9,1.3,1.7$ and $2.0 \mathrm{keV}$. Within the scaling of Fig. 20 no larger energy dependence is seen. The agreement with the full PSF model from (28)in the interval $[0,500]$ arcsec is acceptable but the tails of $p_{\mathrm{G}}^{\mathrm{V}}$ fall off too steeply in comparison with Fig. 18. The energy dependence is weak enough that, at the scale of Fig. 20, the curves $p_{\mathrm{S}}^{\mathrm{G}}$ fall close together. 


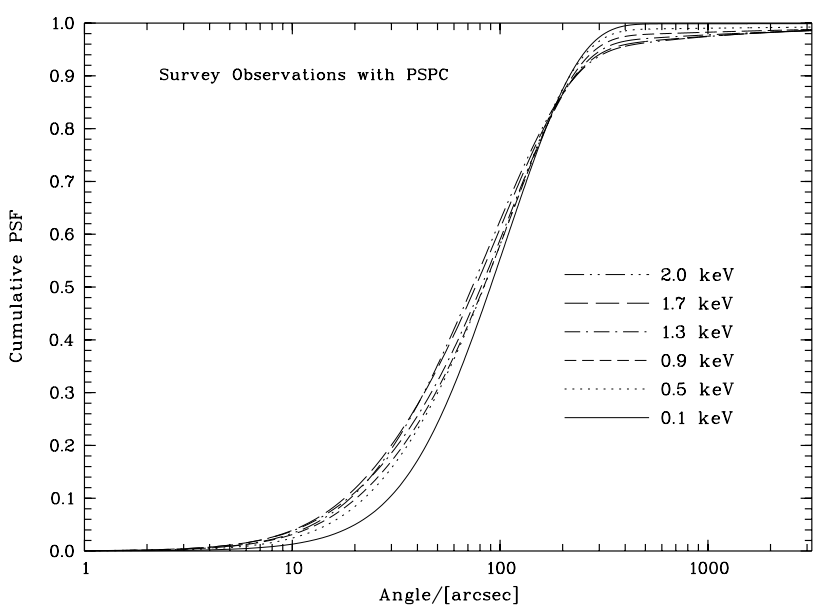

Fig. 19. Cumulative PSPC Survey PSF for $E=0.1,0.5,0.9$, $1.3,1.7,2.0 \mathrm{keV}$

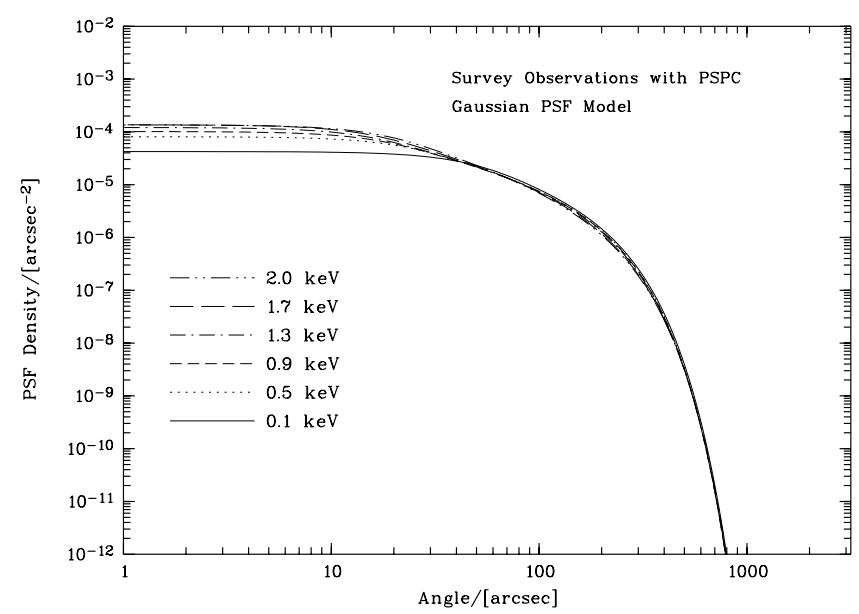

Fig. 20. PSPC survey PSF $p_{\mathrm{S}}^{\mathrm{G}}(r ; E)$ based on the Gauss approximation for $E=0.1,0.5,0.9,1.3,1.7,2 \mathrm{keV}$ for $r \in[1,3600]$ $\operatorname{arcsec}$

The cumulative counter-part of Fig. 20 is shown in Fig. 21. We see that the finer model underlying Fig. 19 leads to about the same weak energy dependence.

\section{Point spread function broadening due to attitude jitter}

During an observation interval $\left[t_{1}, t_{2}\right]$, the instrument's optical axis jitters around a nominal direction due to the behaviour of the attitude control loop. An additional deliberate wobbling motion is usually superimposed in pointed observation mode and corrected for. In the standard ROSAT data analysis, a software correction for the rest wobbling motion and non-perfect attitude data is applied leaving a smaller uncorrected rest wobbling. With or without a rest wobbling the jitter motion remains. The movement of the optical axis manifests itself in the

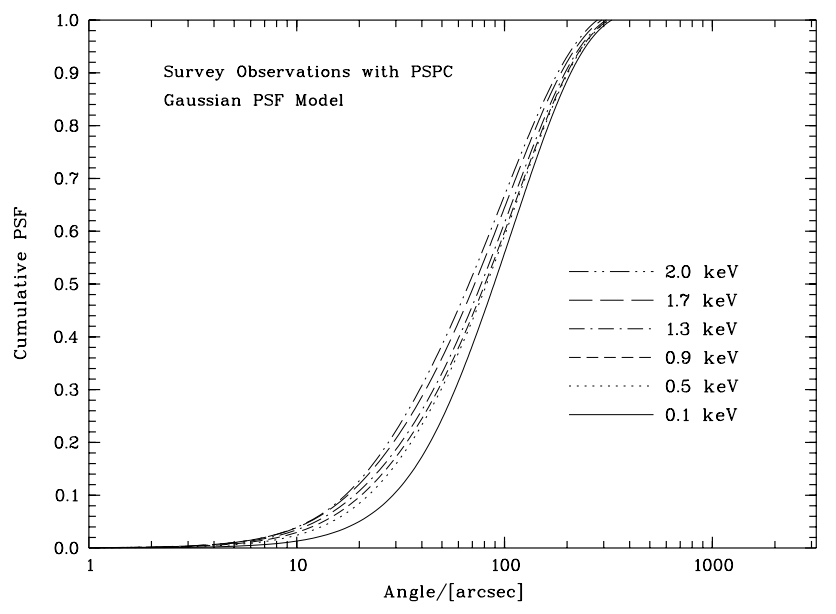

Fig. 21. Cumulative PSPC survey PSF $P_{\mathrm{S}}^{\mathrm{G}}(r ; E)$ based on Gauss approximation for $E=0.1,0.5,0.9,1.3,1.7,2 \mathrm{keV}$ for $r \in[1,3600] \operatorname{arcsec}$

detector plane as a translation of any image point. Consider in the sequel a true source point $s$ along with its related jitter trajectory $s(t)$.

When viewing the composite motion, jitter plus possible wobbling, as an instrument property we are confronted with the problem to determine the PSF under this composite motion. The jitter motion is statistically independent from the infall of photons and from the detector dynamics.

First, we assume a sufficient long observation span $T:=t_{2}-t_{1}$ to the effect that an area element in the detector plane around the hypothetical source position $s$ is traversed often enough by the source trajectory $s(t)$ evolving in time $t, t \in\left[t_{1}, t_{2}\right]$. Under this assumption it is reasonable to assume a probability distribution density $w(x, s)$ for the composite source motion. The probability to find the source in a neighbourhood with area $d x$ around a point $x$ is thus $w(x, s) \mathrm{d} x$. Clearly,

$w(x, s) \geq 0, \quad \int_{\mathbf{R}^{2}} w(x, s) \mathrm{d} x=1$.

In presence of a jitter motion with density from (31) a PSF density $p(r ; E, \epsilon)$ for a steadfast optical axis becomes transformed to

$p_{w}(x, s):=\int_{\mathbf{R}^{2}} w(y, s) p(|x-y| ; E,|y|) \mathrm{d} y$.

Compared with the detector's field of view, the source motion $s(t)$ takes place in a tiny neighbourhood of the nominal source position $s$. This combined with the fact that $p(r ; E, \epsilon)$ varies slowly with respect to the off-axis angle $\epsilon$ allows the approximation $|y| \approx|s|$. Adoption of this approximation casts (31) into the shape

$p_{w}(x, s):=\int_{\mathbf{R}^{2}} w(y, s) p(|x-y| ; E,|s|) \mathrm{d} y$. 
Next, we assume two-dimensional normal distributions for $p$ and $w$ in (33). Remind the following.

Lemma. Let $X_{k}, k=1,2$, be two independent, normally distributed random $n$-vectors with expectations $\mathbf{E}\left(X_{k}\right)=: \mu_{k}$ and $n \times n$ variance-covariance matrices $\operatorname{Var}\left(X_{k}\right)=: V_{k}$. Then the sum $X:=X_{1}+X_{2}$ is again normally distributed with expectation $\mu:=\mu_{1}+\mu_{2}$ and variance matrix $V:=V_{1}+V_{2}$. Thus, the sum $X$ possesses the density

$$
\begin{aligned}
p_{X_{1}+X_{2}}(x):= & \frac{1}{(2 \pi)^{n / 2} \cdot \sqrt{\operatorname{det}\left(V_{1}+V_{2}\right)}} \cdot \\
& \mathrm{e}^{-\frac{1}{2}\left[\left(x-\mu_{1}-\mu_{2}\right)^{\prime}\left(V_{1}+V_{2}\right)^{-1}\left(x-\mu_{1}-\mu_{2}\right)\right]}, x \in \mathbf{R}^{n} .
\end{aligned}
$$

The prime in (34) means transposition.

We neglect the rest wobbling and confine us to plane circular symmetric normal densities $w(x, s):=w(|x-s|)$ with a standard deviation $\sigma_{\text {Att }}>0$ for the attitude motion in

$$
w(r):=\frac{1}{2 \pi \sigma_{\mathrm{Att}}^{2}} \mathrm{e}^{-\frac{1}{2}\left(\frac{r}{\sigma_{\mathrm{Att}}}\right)^{2}}, \quad \sigma_{\mathrm{Att}}>0 .
$$

A standard deviation of $\sigma_{\mathrm{Att}}=3$ arcsec is to be expected for the observation under analysis. A statistical investigation on the variability of this value from observation to observation is not yet carried out.

We apply the above lemma to a circular symmetric normal density as the one in (35) but with standard deviation $\sigma$ in place of $\sigma_{\text {Att }}$ and find the addition law for the considered standard deviation $\sigma$ in (35),

$\sigma\left(\sigma_{\mathrm{Att}}\right):=\sqrt{\sigma^{2}+\sigma_{\mathrm{Att}}^{2}}$

due to the presence of a circular symmetric jitter distribution for the optical axis with standard deviation $\sigma_{\text {Att }}>0$.

Finally, we apply the broadening rule (36) to the normal component in the PSPC having the standard deviation $\sigma(E, \epsilon)$ from $(7)$ as well as to the two normal components in the HRI PSF with standard deviations $\sigma_{1}, \sigma_{2}(\epsilon)$ from (11).

The weights of the remaining PSF components are so small that no further (more difficult) correction efforts are made. This approximation is deemed to be good for the HRI and acceptable for the PSPC. No jitter broadening correction was foreseen for the WFC because a jitter in the order of $\sigma_{\mathrm{Att}}=3 \mathrm{arcsec}$ is negligible in the case of the WFC angular resolution.

Without further information, it cannot be decided whether a positive $\sigma_{\text {Att }}$ is due to an extended celestial source or due the behavior of the observing instrument.

The following Figs. 22 and 23 demonstrate the effect of a PSF correction for the jitter motion and provide also a way to obtain an estimate for the jitter amplitude.

A ROSAT HRI pointed observation of the millisecond pulsar PSR J0437-4715 was performed in 1994 in the usual wobbling mode. A question on source extension could not be decided on the base of this observation (see Becker \& Trümper 1998). Therefore, a re-observation without wobbling on the same object was carried out in 1997. Here, only the jitter motion of the optical axis broadens the HRI PSF. Figure 22 shows the histogram of the observed ring-integrated surface brightness distribution (black dots) with error bars according to Poisson distributions in an 80 arcsec circle centered at the pulsar position. Totally 40 histogram classes of equal width were used to cover the larger concentric disk of radius 120 arcsec.

The observed histogram was compared with the histogram of the HRI PSF taken with $\sigma_{\text {Att }}=0$ arcsec (solid line) on the same radial range $[0,120]$ arcsec. The residual plot suggests already that the observed histogram and the HRI PSF histogram are not in good agreement. The reduced $\chi^{2}$ for $40-1=39$ degrees of freedom amounts to $\chi_{\text {red.,39 }}^{2}:=\chi^{2} / 39=9.078$.

For a significance level of $90 \%$ the critical value of the $\chi^{2}$-distribution with 39 degrees of freedom becomes $\chi_{\text {crit.,39,0.9 }}^{2}=50.660$. This means a critical reduced $\chi^{2}$ value $\chi_{\text {red.,crit.,39,0.9 }}^{2}=1.299<9.078$ which is grossly exceeded by the observed reduced $\chi_{\text {red.,39 }}^{2}$. The rejection of the hypothesis of the equality of the HRI PSF distribution and the pulsar distribution underlying Fig. 23 is thus statistically justified.

An estimate for $\sigma_{\text {Att }}$ was found to be $\hat{\sigma}_{\text {Att }}=2.6$. Figure 23 shows the same observed histogram as in Fig. 22 along with the HRI PSF histogram now for $\sigma_{\mathrm{Att}}=2.6 \mathrm{in}$ place of the former $\sigma_{\mathrm{Att}}=0$. The observed reduced $\chi^{2} \mathrm{di}-$ minishes to $\chi_{\text {red.,38 }}^{2}=0.922$ which is well below the critical value for 38 degrees of freedom $\chi_{\text {red...crit..38 }}^{2}=1.303$. Now, the equality of radial pulsar and HRI PSF distributions cannot be rejected on the $90 \%$ significance level.

\section{Conclusions}

The PSF density and the cumulative PSF of all telescopedetector combinations aboard the ROSAT satellite as used by the EXSAS analysis system (and the standard ROSAT data analysis system) are described here. All these distributions, save those for the WFC, are azimuthally averaged parts of the observed PSFs and depend on the energy $E$ of the infalling photons as well as the off-axis angle $\epsilon$ over the corresponding energy range and the off-axis angle range for each mirror-detector optical unit. The derivation of the survey PSF from the PSF in pointing mode is given. Equally, the broadening of the PSF due to the attitude jitter is modelled and verified by a ROSAT HRI observation of a millisecond pulsar without wobbling.

Acknowledgements. A lot of work went into ground calibrations and in-flight calibrations carried out by many colleagues at the Max-Planck-Institut für extraterrestrische Physik, Garching, Germany, the Astronomisches Institut of the 

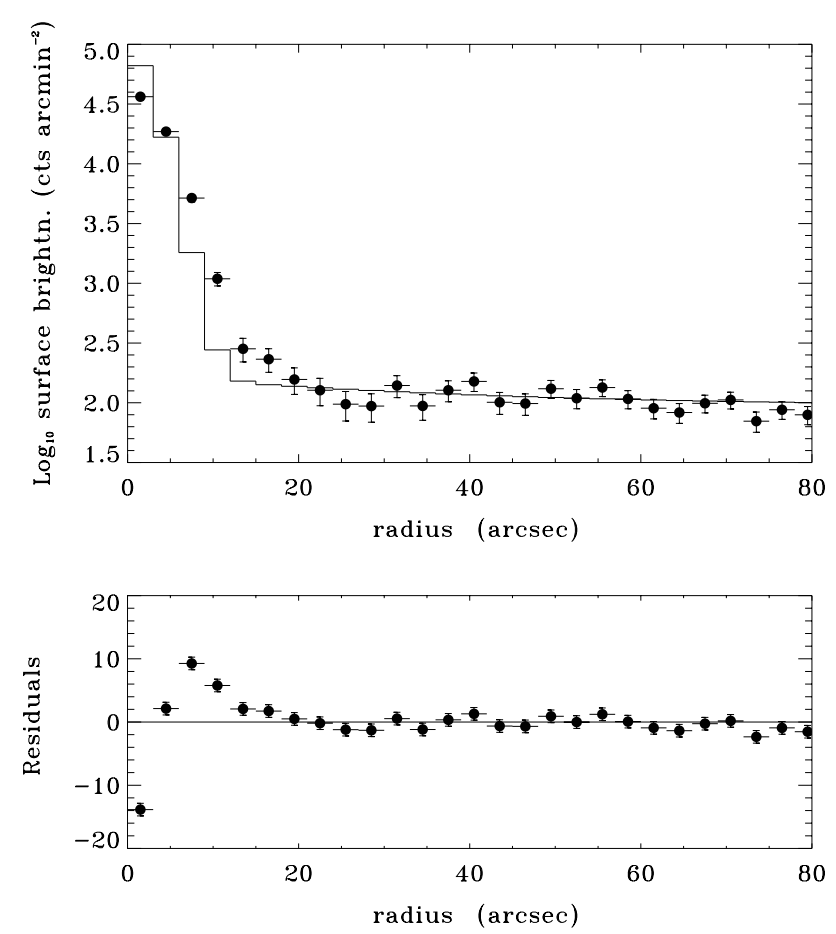

Fig. 22. The radial distribution histograms of the HRI observation of PSR J0437-4715 (black dots) and the HRI PSF distribution histogram (solid line) with $\sigma_{\text {Att }}=0$ in the radial range $[0,80]$ arcsec. 40 equal histogram classes were used in $[0,120]$ arcsec. Centre is the pulsar position

University of Tübingen, Germany, X-ray Astronomy Group of the Department of Physics and Astronomy, University of Leicester, United Kingdom, the United States ROSAT Science Data Center, Harvard University, Smithonian Center for Astrophysics, Cambridge, MA, and the NASA Goddard Space Flight Center, Greenbelt, MD. All of them have contributed to the present knowledge about the mirrors and detectors aboard of ROSAT which provided the base for the implementation of the PSF related software into the EXSAS analysis system. Here is not the place to ascribe the contributions in more detail to persons or groups. In this respect, the interested reader is referred to the related documents below.

It is a pleasure for the author to thank his colleagues P. Predehl and U. Zimmermann from his institute for painstakingly reading the manuscript and for the many constructive comments. Figures 22 and 23 are courtesies of W. Becker from the same institute. The author would also like to thank Frederic Magnard, Institut d'Astrophysique, Paris, for pointing out to him a small difference between Fig. 4 and the corresponding formula (9) in an earlier version.

The ROSAT project has been supported by the German BMFB, DLR, and the Max-Planck society.

\section{Appendix: EXSAS aids for the ROSAT PSFs}

The EXSAS (extended scientific analysis system), see Zimmermann (1998), assists in analyzing ROSAT data sets. It is distributed to several dozens of institutes. Some
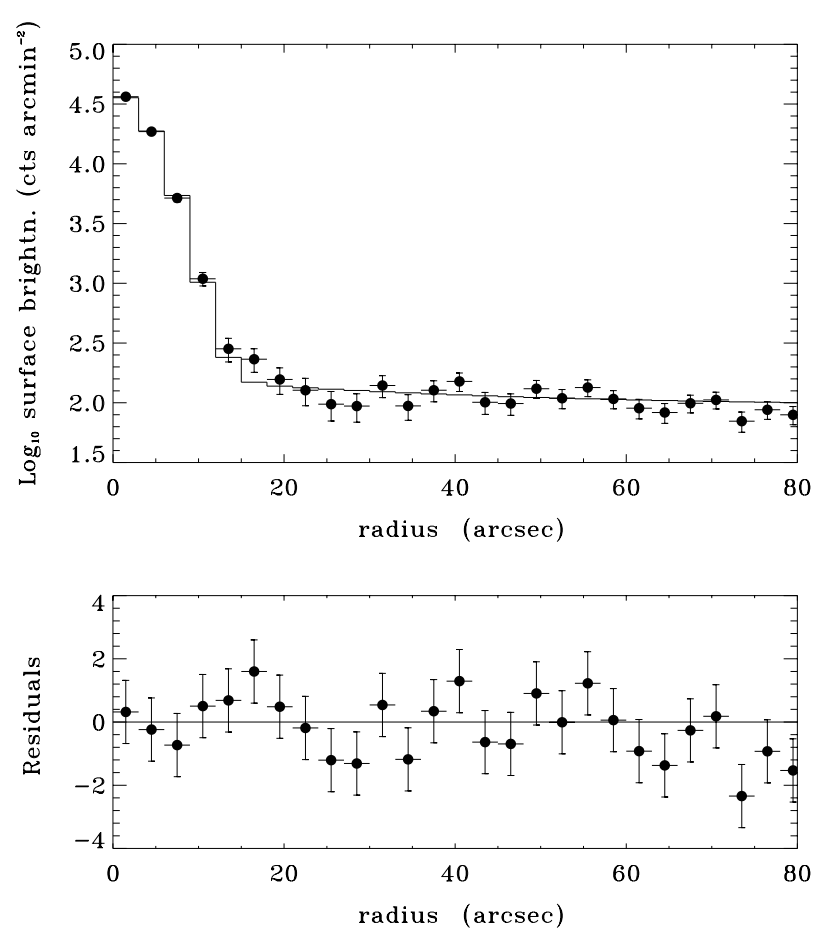

Fig. 23. The radial distribution histograms of the HRI observation of PSR J0437-4715 (black dots) and the HRI PSF distribution histogram (solid line) with $\sigma_{\text {Att }}=2.6$ in the radial range $[0,80]$ arcsec. 40 equal histogram classes were used in $[0,120]$ arcsec. Centre is the pulsar position

commands closely related to point spread functions topics shall be explained here.

The EXSAS command

CALCULATE/PSF

evaluates for the selected ROSAT instrument the density $p(r ; E, \epsilon)$, the cumulative PSF $P(r ; E, \epsilon)$, the FWHM $w(E, \epsilon)$, and the median radius $r_{1 / 2}$. In another evaluation mode, the inverse of the cumulative distribution, i.e. the quantile radius $r_{\mathrm{q}}$ as introduced in Sect. 3, is returned. The median can be calculated using the EXSAS command (37).

Other point spread function related EXSAS commands are

CREATE/PSF,

$M A K E / P S F \_M O D E L \_P R O F I L E$, PLOT/PSF_MODEL_PROFILE.

The first command in (38) returns the PSF in form of an image. The second one calculates tables with PSF density or cumulative values. Those tables can be plotted with the help of the third command. The figures of this paper were made with the last two commands. The EXSAS command

COMPUTE/PSF_OVERLAP 
is a further PSF related command. Assume a given pair of point sources which are close together. Then choose two disk with two radii having the point sources as centres. In one operation mode, the command returns the fraction of photons falling into its surrounding disk and, additionally, the fraction of photons from each source falling into the intersection region of the two disk. The intersection region may be void.

The PSPC point spread function density $p(r ; E, \epsilon)$ is held in table form in

$E X S A S \_C A L: p s f \_p s p c_{\_} \epsilon \epsilon . t b l, \epsilon \epsilon \in\{00,05,10, \cdots, 50\}$,

where $\epsilon \epsilon$ stand for the two digits of the off-axis angle $\epsilon$ in arcmin. Each of the tables (40) has 21 columns. Besides the column for $r=1(1) 3600$ arcsec, labelled $A N G L E$, are a further 20 columns for the energies $E=0.1,0.2, \cdots$, $2.0 \mathrm{keV}$, labelled RCTS $0 D 1$ to $R C T S 2 D 0$. The first table in (40) is the on-axis table, $\epsilon=0$.

Analogously, tables for the HRI point spread function $p(r ; E, \epsilon)$ are held in

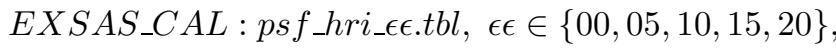

EXSAS_CAL:psf_hri_g_t $\epsilon . t b l, \epsilon \epsilon \in\{00,05,10,15,20\}$,

The table format is the same as the one for the PSPC. The last group of tables in (41) tabulates a Gauss approximation to the full PSF model.

The internet access to the most important ROSAT documents and to the HRI calibration report is possible via the two addresses

http://wave.xray.mpe.mpg.de/rosat

http://hea - www.harvard.edu/rosat/

rsdc_www/HRI_CAL_REPORT/hri.html

Presently, it cannot be said how long the post mortem public access (42) will be maintained.

\section{References}

Aschenbach B., 1988, Appl. Opt. 27, 1404

Barstow M.A., Sansom A.E., 1990, in: SPIE, Vol. 1344, EUV,
X-ray, and Gamma-ray instrumentation for astronomy, Siegmund O.W., Hudson H.S. (eds.), San Diego, p. 244

Becker W., Trümper J., 1999, A\&A 341, 803

Brunner H., Kreysing H.C., 1993, ROSAT/WFC Observer Notes 93/1, Rev. 1, Universität Tübingen, Germany (Report)

David L.P., Harnden Jr. F.R., Kearns K.E., Zombeck M.V., 1999, The ROSAT High Resolution Imager (HRI) Calibration Report, U.S. ROSAT Data Center/SAO (revision June 1999)

Hasinger G., Turner T.J., George I.M., Boese G., 1992, ROSAT PSPC The On-Axis point spread function: in-flight comparison with the PANTER results, Legacy 2, Nov., 77

Hasinger G., Turner T.J., George I.M., Boese G., 1993, ROSAT PSPC The On-Axis point spread function: inflight comparison with the PANTER results, Addendum: High Signal-to-Noise In-Flight Data; Legacy 3, May, 46

Hasinger G., Boese G., Predehl P., et al., 1994, ROSAT PSPC Off-Axis Point Spread Function, Legacy 4, February, 40

King I.R., 1983, PASP 95, 163

Moffat A.F.J., 1969, A\&A 3, 455

Sansom A.E., 1990, ROSAT WFC PSF, University of Leicester, UK, (Report)

Sansom A.E., 1991, ROSAT WFC PSF - Analysis of In-orbit Calibration and Survey Data, University of Leicester, UK, (Report)

Trümper J., 1990, ROSAT: Call for Proposals for the first observing period November 1990 - April 1991, Technical Appendix. Max-Planck-Institut für extraterrestrische Physik, Garching

Trümper J., 1991, ROSAT: Call for Proposals for the second observing period, August 1991 - February 1992, Technical Appendix. Max-Planck-Institut für extraterrestrische Physik, Garching

Voges W., Aschenbach B., Boller Th., et al., 1999, A\&A 349, 389

Wells A., Abbey A.F., Barstow M.A., et al., 1990, in SPIE Vol. 1344 EUV, X-ray, and Gamma-ray instrumentation for astronomy, Siegmund O.W., Hudson H.S. (eds.), San Diego, p. 230

Willingale R., 1988, Appl. Opt. 27, 1423

Zimmermann U., Boese G., Becker W., et al., 1998, EXSAS User's Guide: Extended Scientific Analysis System to Evaluate Data from the Astronomical X-ray Satellite ROSAT. Max-Planck-Institut für extraterrestrische Physik, Garching bei München, Germany 